\title{
Investigation of Anti-Phase Asymmetric Quiet Rotor Technology
}

\author{
Nicholas B. Cramer* \\ Stinger Ghaarian Technologies, Inc., Moffett Field, CA 94035 \\ Nhan T. Nguyen ${ }^{\dagger}$ \\ NASA Ames Research Center, Moffett Field, CA 94035 \\ Sarah R. Hernandez ${ }^{\ddagger}$ \\ JACOBS, Moffett Field, CA 94035 \\ Bruce L. Storms ${ }^{\S}$ \\ NASA Ames Research Center, Moffett Field, CA 94035
}

\begin{abstract}
The future of urban air mobility has a well-known tall pole challenge in the form of community acceptance which largely comes from the noise. This paper presents a proposed antiphase rotor technology that could reduce noise sources such as blade vortex interaction noise. The anti-phase rotor technology includes a rotor design with various anti-phase alternating trailing edge patterns and a rotor design with an asymmetric blade tip. Four small-scale antiphase rotors are fabricated by $3 \mathrm{D}$ printing for acoustic measurements conducted in a low-speed open-circuit wind tunnel to assess the effectiveness of the proposed anti-phase rotor technology. Preliminary test results appear to be promising and indicate that the anti-phase rotor designs could be a practical means of reducing blade vortex interactions and noise. The four tested antiphase rotor designs have peak acoustic performance depending on the RPM and thrust which suggests improved performance through design optimization could be achieved for specific mission requirements.
\end{abstract}

\section{Nomenclature}

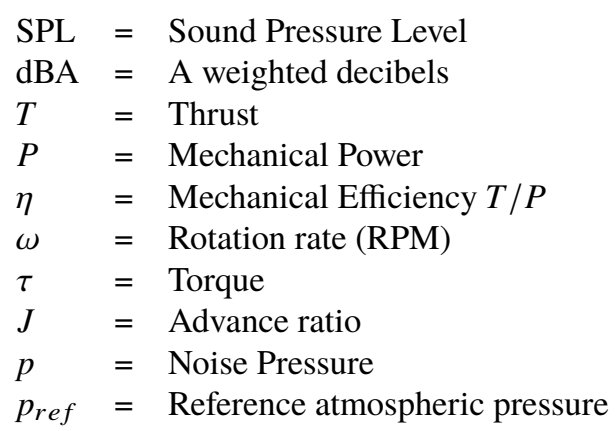

\section{Introduction}

T RBAn Urban air mobility(UAM) concepts have been proposed as an ability to dramatically decrease the door to door travel time for short to mid-distance trips (20-200 miles)[1]. The technological development of distributed electric propulsion has enabled multi-rotor designs that are capable of reducing the cost of vertical take-off and landing flight by

\footnotetext{
*Research Engineer, Intellegent Systems Division, NASA Ames Research Center,nicholas.b.cramer@nasa.gov

${ }^{\dagger}$ Research Scientist, Intelligent Systems Division, nhan.t.nguyen@nasa.gov, AIAA Associate Fellow

$\doteqdot$ Associate Engineer, Experimental Aero-Physics Branch

${ }^{\S}$ Researcher, Experimental Aero-Physics Branch
} 
reducing the complexity of design, operational cost via fuel savings, and fixed operating cost via insurance reductions. [2] These cost reductions could go a long way to reaching the necessary financial conditions for a market to develop.

Due to market conditions and geographic limitations Silicon Valley has been proposed as an ideal early market for UAM operations. The analysis has shown that much like helicopters, UAM noise is one of the primary technical challenges that need to be addressed in order to have full adoption. Antcliff et al. proposed an acceptable noise margin of $65 \mathrm{dBA}$ at a distance of $250 \mathrm{ft}$ which represents the acceptable noise level of large trucks to domestic housing. [3] The burgeoning industry has adopted this criterion [4].

The majority of UAM noise comes from the aircraft's rotors. Rotor noise is a complex problem with many different potential sources through the noise sources are generally grouped into a few categories:

- Loading Noise: This is the noise that is generated from the steady state thrust of the propeller[5].

- Vortex Noise: The vortex noise is a dominant broadband effect that is the result of the trailing edge and tip shedding vortices[6].

- Rotational Noise: This noise is associated with the rotational speed and is a multiple of the blade pass frequency[5, 6].

- Blade Vortex Interaction: Blade vortex interaction (BVI) is the interaction of the trailing vortices with another rotor blade[7].

Most of the research has focused on the reduction of vortex or BVI noise. The attempts to address vortex noise are typically made by altering the tip or the trailing edge of the blades. Brocklehurst[8] and Yung [9] provide reviews of the various forms of tip alterations that address either vortex or BVI noise, most designs focus either on altering the location of the vortex core or creating multiple smaller vortices. Other approaches to reducing the vortex noise include varying the trailing edge which primarily reduced tonal noise, but it also thought to address broadband noise. The most common variation is a serrated trailing edge, though it comes in various forms. Geiger et al. proposed a chord-wise serrated trailing edge to increase the decay rate of the blade wake, while Oerlemans et al. proposed a similar concept but in a spanwise orientation on a wind tunnel turbine blade. Both projects were able to demonstrate noise tonal noise reduction. The serrated designs are not the only alterations to the trailing edge that have shown acoustic benefits, the use of a wavy trailing edge in for wind turbine application was shown to have some benefits in CFD simulations[10]. Additional serrated trailing edge alterations have also shown reductions of BVI noise in CFD simulations. Outside of vortex noise, the next most common noise to be addressed is BVI. Some other design approaches to address BVI included blades bent to disrupt the tip vortices[11] and blade dissimilarities that perform a similar function[12]. These designs have all been passive mechanisms, but there have even been proposed solutions for active twist mechanisms to reduce the vortex noise actively, demonstrated in simulation[13] .

\section{Anti-Phase Rotor Concepts}

Almost all existing rotor noise suppression concepts employ an identical rotor design in one form or another as a standard industry practice for ease of manufacturing. However, one attribute of an identical rotor design is that the shed vortices from a preceding rotor blade are always reinforced by a following rotor blade. This effectively causes a harmonic reinforcement of the blade shed vortices without any means of altering the shed vortex structure in a favorable way so as to reduce the BVI noise in an identical blade design. Turbomachinery designers have long recognized the significance of breaking up the harmonic reinforcement in rotor blade designs as a mean of preventing a resonant vibration or reducing the forcing function in a blade row. One common practice in turbomachinery design is to employ a split-half stator design with unequal blade counts in the upper half and lower half of a stator [14]. This design effectively disrupts the harmonic reinforcement of the stator wakes as seen by the passing rotor blades, thereby reducing the vibration of the rotor blades due to blade passing frequencies.

Another example is the method of circumferentially varying a stator stagger angle (stagger angle equals $90^{\circ}$ minus blade pitch angle) to alter the blade forcing function. One of the authors had applied this method in a low-speed axial-flow compressor in the NASA Ames 11-Foot-By-11-Foot Transonic Wind Tunnel to alter the upstream wake interaction on the rotor blade forcing function [15]. Figure 1 illustrates the three-stage compressor in the NASA Ames 11-Foot-By-11-Foot Transonic Wind Tunnel. By circumferentially varying the blade stagger angle of the exit guide vanes in such a way that diverts the flow around five downstream support struts, the wake upstream propagation due to the potential flow field created by the five support struts is altered in a favorable manner that results in a reduced amplitude of the 5/rev forcing function on the upstream rotor blades, thus effectively attenuating the 5/rev resonant vibration of these rotor blades. 


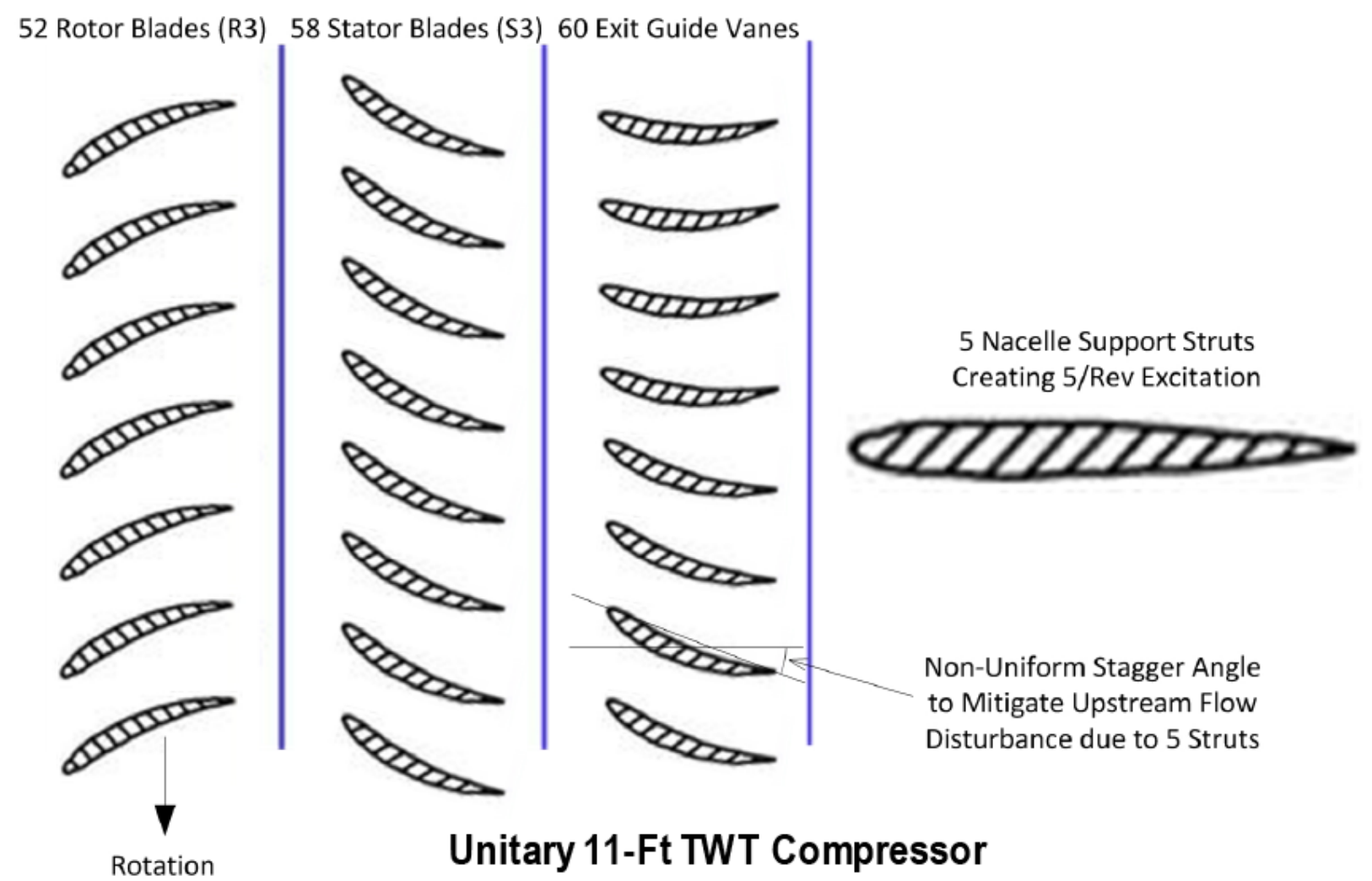

Fig. 1 Non-Uniform Stator Stagger Angle in Three-Stage Compressor in NASA Ames 11-Foot-By-11-Foot Transonic Wind Tunnel

Inspired by the vibration reduction benefit of the circumferentially varying blade stagger method in the NASA Ames 11-Foot-By-11-Foot Transonic Wind Tunnel, this author hypothesizes that if the harmonic reinforcement of the blade vortex structure could somehow be disrupted, acoustic attenuation benefits could potentially be realized. This leads to a number of acoustic reduction concepts that have been proposed to NASA Convergent Aeronautics Solutions (CAS) project in the past and most recently in 2017 [16]. Collectively, these proposed concepts are referred to as anti-phase rotor concepts. The experimental results reported in this paper are from a funded activity by the CAS project that briefly lasted from October 2017 to March 2018. A follow-on effort is currently under way and is funded by NASA Ames Center Innovation Funds (CIF) program [17]. This follow-on effort will investigate acoustic benefits in CFD simulations as well as conduct an acoustic experiment in an anechoic chamber at Pennsylvania State University (PSU).

Figure 2 illustrates the proposed anti-phase rotor noise reduction concepts. One anti-phase concept is embodied by a continuous trailing edge shape modified in an anti-phase alternating pattern from blade to blade so as to prevent harmonic reinforcement of the blade vortex structure that could lead to an acoustic reduction. Figure 2 further illustrates two concepts of a two-bladed rotor employing an anti-phase alternating trailing edge pattern. On one blade, the anti-phase trailing edge pattern is $180^{\circ}$ out of phase with respect to the anti-phase trailing edge pattern on the other blade. This is further illustrated in Figure 2 showing the anti-phase trailing edge patterns of the preceding blade and following blade being out of phase, for example, the trailing edge in a downward deflection on the preceding blade but in an upward deflection on the following blade at the same radial position. In another embodiment, the anti-phase trailing edge pattern does not follow the exact $180^{\circ}$ out-of-phase rule. This is shown by the first line and third line illustrating the anti-phase trailing edge pattern in Figure $2 \mathrm{~B}$. To break up the harmonic reinforcement along the blade span, the anti-phase pattern is applied over several segments of the trailing edge with a constant length or varying length from blade to blade. 


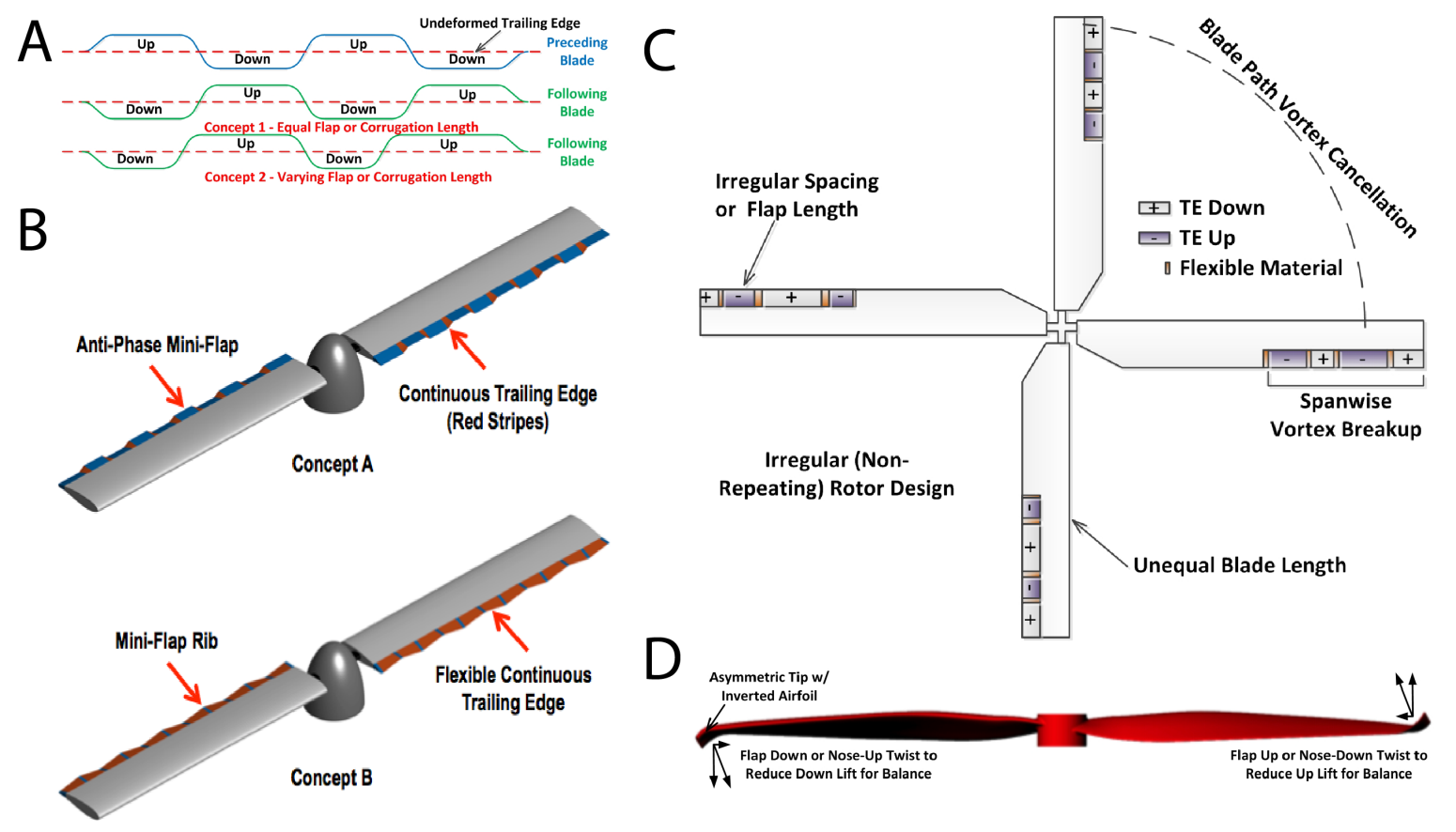

Fig. 2 The anti-phase concept is displayed in figure $A$ with the alternating trailing edge for the leading and following blades including irregular trailing edge shapes. Figures $B$ and $C$ display variations of active anti-phase trailing edge designs with continuous trailing edges utilizing flexible materials between the flaps. An alternative anti-phase design uses anti-symmetric blade tip to move the vortex centers out of plane with each other.

The anti-phase trailing edge can be accomplished by either a passive design or active design. A passive design has the advantage of design simplicity and would target a minimum noise impact requirement during hover or descent. The disadvantage of a passive design is the negative impact on the aerodynamic performance of the rotor which could result in increased blade losses, hence increased rotor power. An active design provides more operational flexibility than a passive design. The active design would utilize a series of anti-phase mini-flaps or ribs with a continuous trailing edge feature [17, 18] to provide a mechanism for adjusting the anti-phase trailing edge pattern as needed or restoring the trailing edge to a conventional undeformed state for improved rotor performance during cruise. The continuous trailing edge in an active design could be enabled by a flexible transition section in between the mini-flaps or a flexible skin over the entire trailing edge surface with ribs. Other embodiments are possible. The disadvantage of an active design is the design complexity that could lead to an increase in weight and power for actuation systems. In addition, reliability is another operational factor that could negate some benefits of an active design.

Figure $2 \mathrm{C}$ illustrates a four-bladed rotor that employs anti-phase rotor blades. The anti-phase rotor blades are all dissimilar and can have unequal blade length. This could provide another source for noise reduction.

Because of the nature of the blade loading with the increased tangential velocity toward the rotor blade tip, the tip vortices can be a significant noise source. Swept anhedral tip treatments are effective in reducing acoustic signatures for transonic rotors. The anti-phase rotor concept can be extended to an anti-phase asymmetric blade tip design as shown in Fig. 2D. This concept involves the blade tip of one blade curved in an upward orientation while the blade tip of the other blade curved in a downward position. If the airfoil layout of the rotor blade continues into the curved blade tip regions, a radial force imbalance would result and would cause operational issues. One way to reduce the radial force imbalance due to the asymmetric blade tip design is to employ an inverted airfoil on one of the blade tips. A typical airfoil section with a positive camber is employed everywhere on the main blade surface. This airfoil is transitioned to an airfoil with zero camber near the blade tip region and a negative camber in the blade tip region.

The mechanisms of noise reduction of the anti-phase rotor concepts could be explained by Fig. ??. A 2D rotor CFD simulation is performed using STAR-CCM+. Two rotor airfoils at a given radial station are modeled using a periodic boundary condition at the inlet of the preceding rotor airfoil with a downward deflected trailing edge and at the exit of the following rotor airfoil with an upward deflected trailing edge, as shown in Figs. $3 \mathrm{~B}$ and $3 \mathrm{C}$. The velocity contour plot of the CFD simulation is shown in Fig. $3 \mathrm{C}$. It can be clearly seen that anti-phase rotor design creates a non-planar 
wake that effectively makes the anti-phase rotor design to exhibit a similar wake behavior as a stacked co-rotating rotor design which has recently been proposed by Uber as a means for rotor noise suppression [19]. Figure 3B shows a wake with an increased strength shed off the preceding airfoil while the following blade sheds off a much weaker wake. Because of the downward deflected trailing edge of the preceding airfoil, its wake completely bypasses the following blade instead of impinging on the following blade. The two wakes of unequal strength can be seen to be non-interacting, thereby reducing the blade vortex interaction. Figure 3 A illustrates the non-interacting downwash and upwash created by the downward and upward deflected trailing edges of the preceding and following blades, respectively.
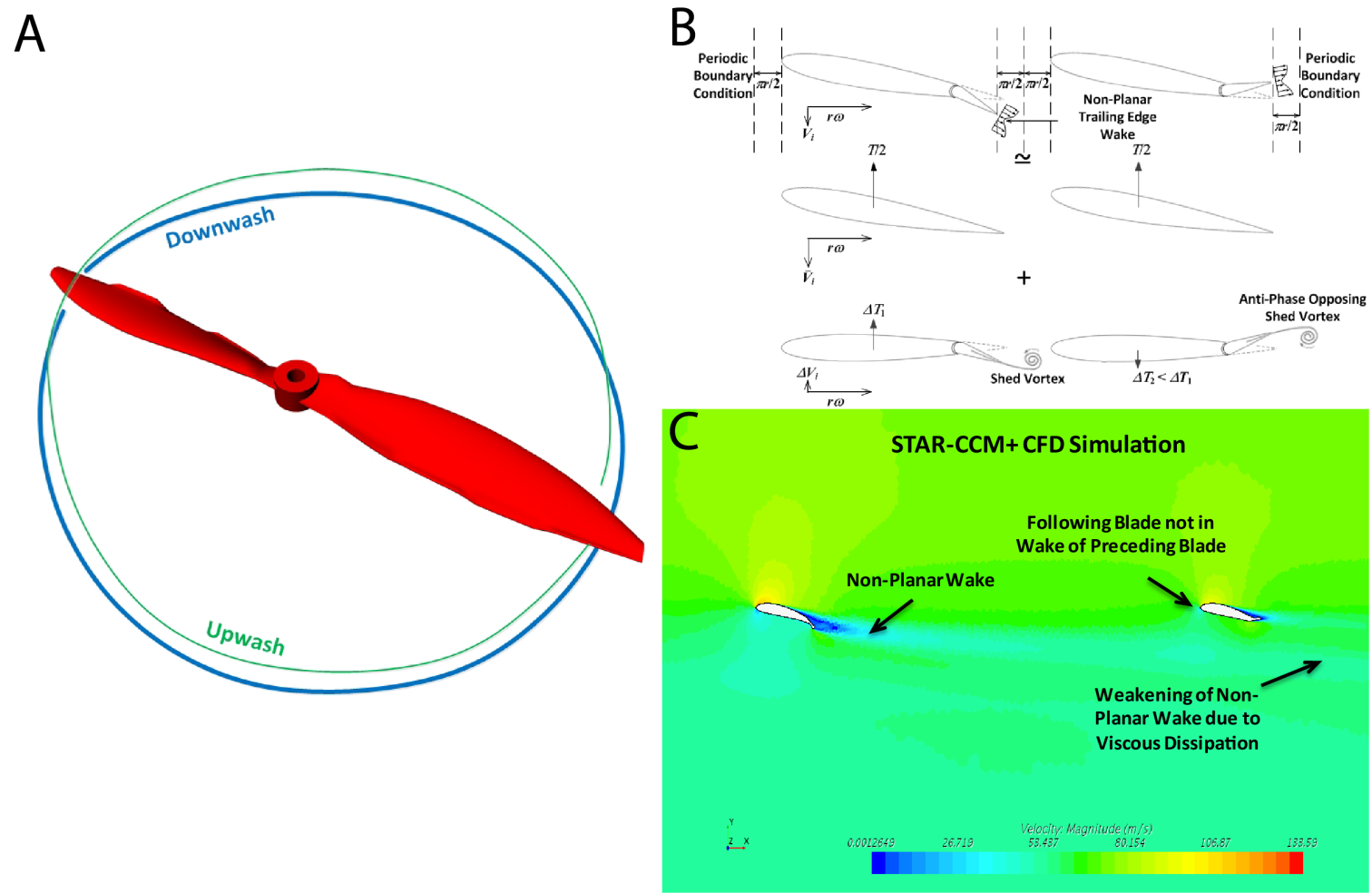

Fig. 3 Proposed Anti-Phase Rotor Noise Reduction Concepts

Another possible mechanism of noise reduction is the lift creation of a downward deflected trailing edge in comparison to that of an upward deflected trailing edge. An airfoil tends to perform more efficiently with a downward deflected trailing edge up to a certain deflection than with an upward deflected trailing edge. The $L / D$ ratio tends to increase as the trailing edge is deflected downward to a certain value. Downward deflected trailing edge has been used as a mechanism for drag reduction for fixed-wing aircraft [20]. As the trailing edge deflects upward, the "kink" in the lower surface could induce an early flow separation that effectively reduces the upwash angle of the flow exiting the trailing edge. The net effect is that for the same downward and upward deflection of the trailing edge, the incremental up-lift created by the downward deflected trailing edge is greater than the incremental down-lift created by the upward deflected trailing edge. This results in a net overall increase in thrust at the same rotor speed. Conversely, the rotor speed can be reduced to achieve the same amount of thrust, thereby lowering the tonal noise generated by the blade passing frequency.

The mechanism of noise reduction of the asymmetric blade tip design could be explained by Fig. 4 . A high-fidelity CFD simulation of the asymmetric blade tip design is performed using LAVA, a NASA Ames high-fidelity CFD code. The vorticity contour plot of the asymmetric blade tip design is compared to that of the corresponding conventional straight blade design. It may be apparent from Fig. 4 that the vorticity at the blade tip of the conventional straight blade design has a higher accumulation than that of the asymmetric blade tip design, which could suggest that the reduction in the harmonic reinforcement of the blade tip vortex structure could be a possible mechanism of noise reduction of the asymmetric blade tip design. 


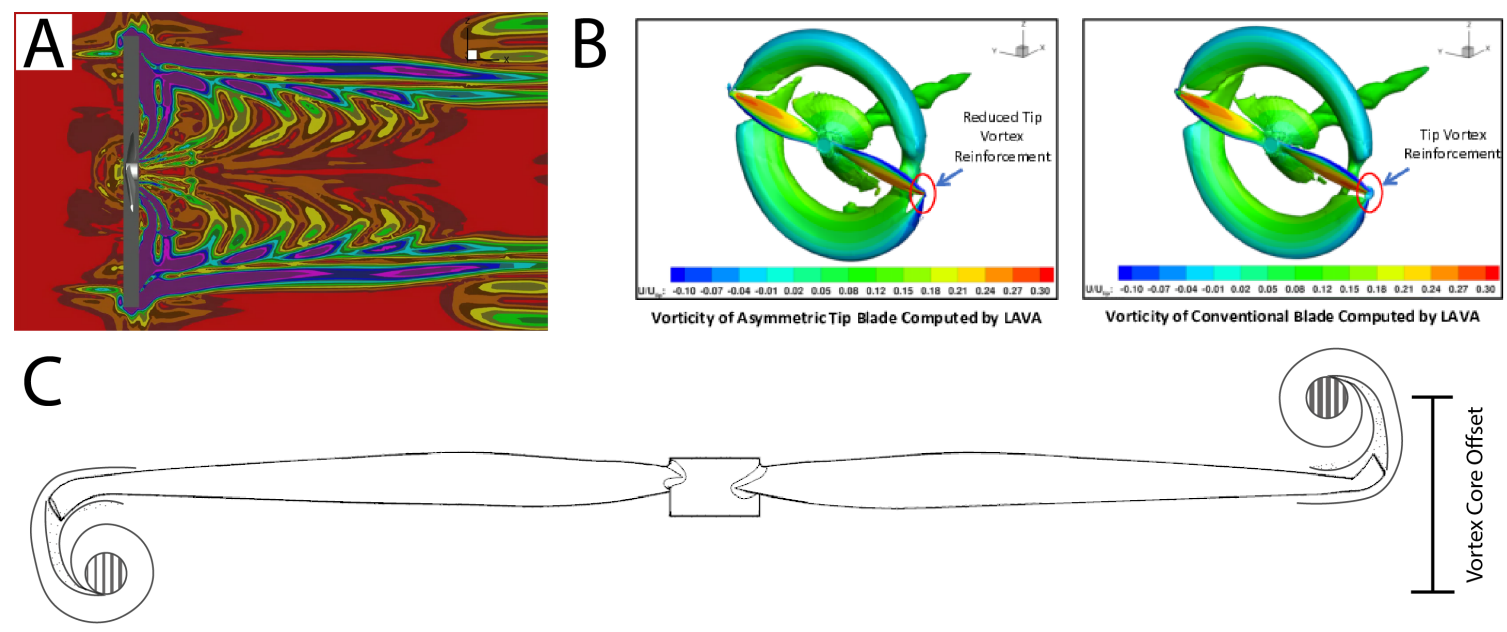

Fig. 4 Proposed Anti-Phase Rotor Noise Reduction Concepts

This paper is organized with the Anti-phase design and experimental set-up in Section IIIffollowed by the experimental results in Section IV] which is split into the mechanical results in Section IV.A and acoustic results in Section IV.B. finally the conclusions and future work are covered in Section $\mathrm{V}$.

\section{Design and Experimental Set-up}

In this paper, we demonstrate four different passive anti-phase configurations: an asymmetric blade tip which is also referred to as "winglet" and three different anti-phase trailing edge patterns with a corrugated shape, a sine shape, and an adjusted sine shape, as shown in Fig. 5 the details of which will be highlighted in a later section. We used an APC 8X3.8 [21] propeller made by Advanced Precision Composites that has a 3.8 in pitch and has an 8 in diameter as the nominal propeller. We took the geometry of the APC 8X3.8 from the UIUC Propeller Database[22]. The thickness of the propeller was doubled for structural purposes to allow for it to be 3D printed and the airfoils used were NACA 2412. There were four anti-phase asymmetric designs the sine wave, adjusted sine wave, corrugated, and asymmetric winglet which are detailed in Table 1 . In each of the trailing edge, designs have an alternating pattern between the two blades intended to alter the planar wake shedding and reduce the constructive interference.
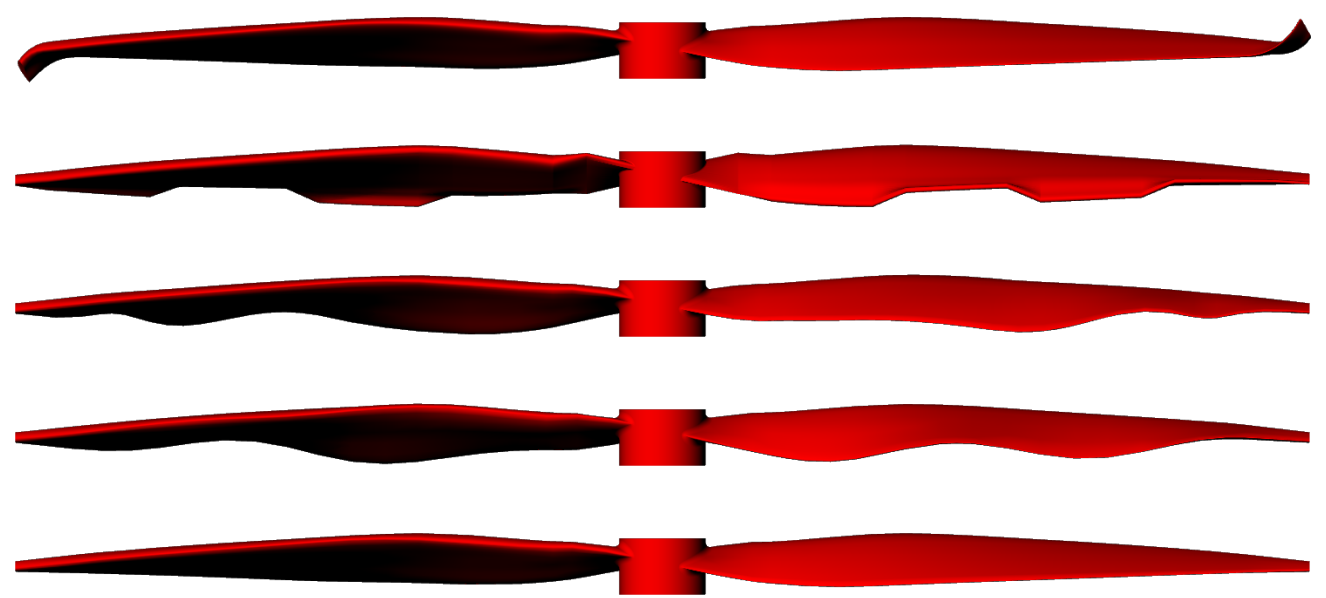

Fig. 5 The bottom propeller blade is the traditional trailing edge blade that the others above it are derived from. The trailing edge configurations going from bottom to top are adjusted sine, sine, corrugated, and asymmetric winglet. 
The three trailing edge patterns were intended to test the effectiveness of different patterns and their ability to maintain/increase performance while reducing noise. All of them were deformed to the same maximum $15^{\circ}$ trailing edge deflection starting from $3 / 4$ chord point. The result was that the corrugated trailing edge had the most area with the maximum trailing edge variation between the leading and following blades a relative difference of $\Delta 30^{\circ}$. We expect that this would result in the substantial planar wake separation. Though the corrugated trailing edge had sharp transitions between its sections that we expected would induce more vortex shedding and an increase in noise. To compare to the impact on those transitions a smooth sine wave with the same magnitude on the trailing edge was produced and the adjusted sine wave which has a more significant portion of the blade deflected but at the lower speed inboard section provides a comparison for the significance of the spatial spanwise inspection on noise.

The final anti-phase design was the asymmetric winglet. The winglet took the last eighth of the blade and rotated up at a $45^{\circ}$ angle along a line from the leading edge at $7 / 8$ of the blade to the trailing edge at $13 / 16$ of the blade, and the opposite was done on the other blade. This extends the ideas of [23] to reduce the interaction between tip vortex and blade-vortex through the use of winglets by further distancing the tip wakes from each other and encouraging destructive interference at specific frequencies.

\begin{tabular}{|c|c|c|c|}
\hline Trailing Edge & \multicolumn{2}{|c|}{ Trailing Edge Displacement } & Description \\
\hline Sine Wave & \multicolumn{2}{|l|}{$15 \sin \left(\frac{4}{b} \pi r\right)$} & $\begin{array}{l}\text { Alternating sine wave along the trailing edge } \\
\text { with magnitude of } 15^{\circ} \text { from the } \frac{3}{4} \text { chord } \\
\text { point for two periods from root to tip }\end{array}$ \\
\hline Adjusted Sine Wave & \multicolumn{2}{|c|}{$\begin{array}{ll}15 \sin \left(\frac{8}{3 b} \pi r\right) & 0>x \leq \frac{3}{8} b \\
-15 \sin \left(\frac{16}{5 b} \pi r-\frac{3}{8} b\right) & \frac{3}{8} b>r>\frac{11}{16} b \\
15 \sin \left(\frac{16}{3 b} \pi r-\frac{11}{16} b\right) & \frac{11}{16} b>r>\frac{7}{8} b \\
-15 \sin \left(\frac{4}{b} \pi r-\frac{7}{8} b\right) & \frac{7}{8} b>r>b\end{array}$} & $\begin{array}{l}\text { Alternating piece-wise sine wave along the } \\
\text { trailing edge with magnitude of } 15^{\circ} \text { from } \\
\text { the } \frac{3}{4} \text { chord point for two periods from root } \\
\text { to tip }\end{array}$ \\
\hline Corrugated & \multicolumn{2}{|c|}{$\begin{array}{ll}-15 & 0>r \leq 0.25 b \\
15 & 0.25 b>r>0.5 b \\
-15 & 0.5 b>r \leq 0.75 b \\
15 & 0.75 b>r>b\end{array}$} & $\begin{array}{l}\text { Alternating } 15^{\circ} \text { trailing edge displacement } \\
\text { from the } \frac{3}{4} \text { chord point }\end{array}$ \\
\hline Asymmetric Winglet & \multicolumn{2}{|l|}{ N/A } & $\begin{array}{l}\text { Tip winglet turned up on one side and down } \\
\text { on the other }\end{array}$ \\
\hline
\end{tabular}

Table 1 Table containing the description of the design variations for the anti-phase designs where $b$ is the half span of the blade and $r$ as the radial position along the span. The opposite sine for each of the trailing edge displacements would be applied to the other blade.

We performed the experiments in the Life Saver tunnel at NASA Ames Fluid Mechanics Laboratory (FML). The Life Saver tunnel is a low-speed open-circuit wind tunnel with a $1 \mathrm{ft}$ by $1 \mathrm{ft}$ test section and is shown in Fig. 61A. The acoustic measurement equipment was not able to fit into the tunnel test section so the experiment was set-up at the exit of the tunnel and a flow straightener wire-mesh was added at the exit, the nominal set-up is shown in Fig. 6 C. This resulted in a 24 -inch by 48 -inch effective test section with maximum airspeed of $40 \mathrm{ft} / \mathrm{s}$. As Fig. 6 shows the microphone array was placed at three different angles during the testing, $60^{\circ}, 90^{\circ}$, and $120^{\circ}$. The $90^{\circ}$ configuration was 37 inches away from the center of the propeller while the $60^{\circ}$ and $90^{\circ}$ configurations were 42.42 -inch. The acoustic data from the microphones was processed using imageJ[24], a Java-based image processing program, and other scripts developed by FML. The microphone array used was a 32-inch Boeing Active Flow Control (AFC) rudder array plate with 24 embedded microphones. The propeller was controlled, and thrust, torque, voltage, current, RPM, and acceleration were measured using the Series 1580 dynamometer and thrust stand made by RCbenchmark[25] which is shown with a propeller mounted on if in Fig. 6 B.

There were two types of experiments performed, constant RPM and constant thrust. Both types of experiments were controlled with proportional-integral (PI) controller. The constant thrust experiments additionally had a moving average filter to smooth out the load cell readings before they were used in the controller. The controller data collection was 
implemented in javascript using the RCbenchmark libraries.

Figure 6P shows the designation of the experimental conditions for descent, hover and ascent. The configurations for descent, hover, and ascent all assume that the observer will be $60^{\circ}$ from negative thrust direction. This would place the observer on the ground while the vehicle is flying. The descent configuration has the thrust in the same direction as the wind which would result in the BVI mentioned in the introduction, whereas the ascent has wind going in the opposite direction.

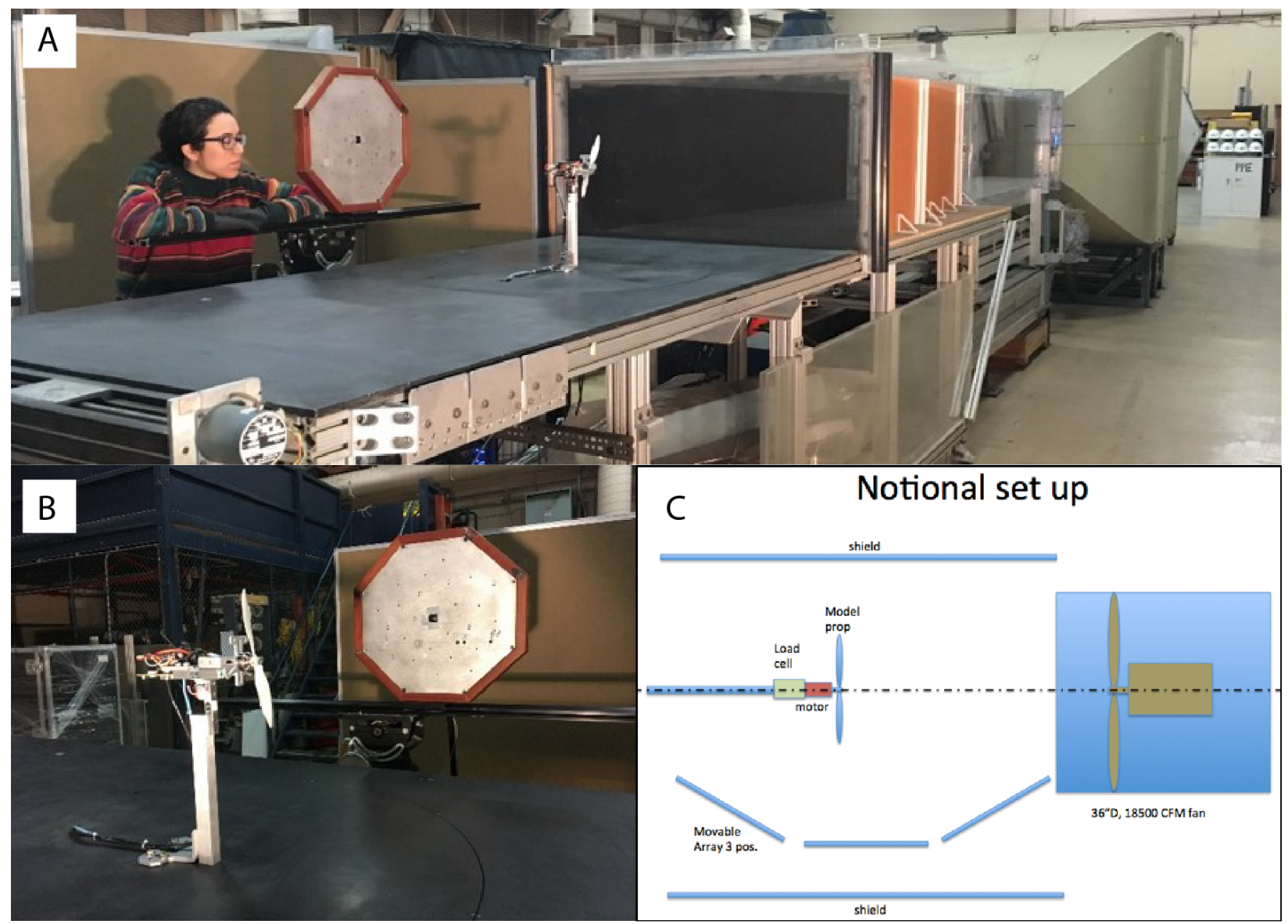

D Descent

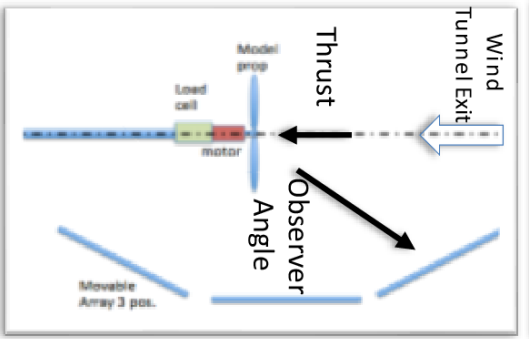

Hover

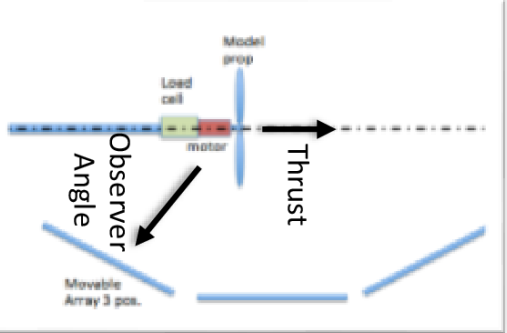

Ascent

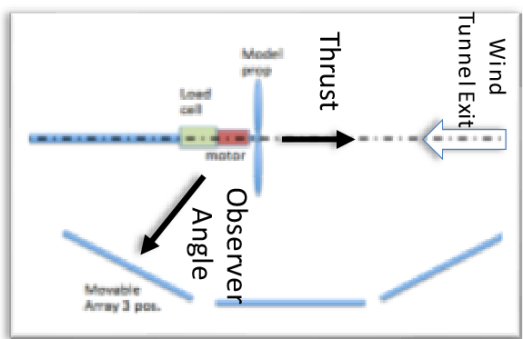

Fig. 6 Figure A shows the wind tunnel, microphone, array and propeller set-up. Figure $B$ is showing the propeller set-up closely whereas $C$ is the notional set-up. Figure $D$ is demonstrating the three different test scenarios for ascent, descent, and hover.

\section{Results and Discussion}

The experimental results take the form of two primary sets, mechanical results, and acoustic results. We focused primarily on the acoustic advantages of the anti-phase trailing edge design, but it is still necessary to be cognizant of the 
performance. If a design is quiet but unable to produce the necessary thrust, then it is not a viable solution. We start by demonstrating that the design variations we are proposing are viable rotor designs from a performance sense then move to the acoustic analysis.

\section{A. Test Results}

The anti-phase designs presented in Section [II] have equal and opposite trailing edge characteristics between each blade. We expect that because of that the variation of thrust between each design and the straight blade nominal design should be minimal. Figure $7 \mathrm{~A}$ shows that the thrust variation is relatively small but that the anti-phase designs show a consistent increase in thrust at a given RPM over the whole range of RPMs in the hover condition. This steady increase in thrust is likely due to the mechanism as explained in the previous section. Figure7 7 is the thrust in ascent configuration at various airspeeds. We see that the general trend from Fig. 7f A continue to hold with the winglet being the sole exception. Each bar in Fig. 7 $\mathrm{B}$ and $\mathrm{C}$ are the averages of three runs at the given wind speed. We can also note that the sine and corrugated trailing edge adaptations have the largest increase in thrust at 4,500 RPM“. This is likely due to the same mechanism as explained in the previous section coupled with the larger surface area of the corrigated trailing edge compared to the surface areas of the sine and adjusted sine trailing edges as well as the asymmetric blade tip design.
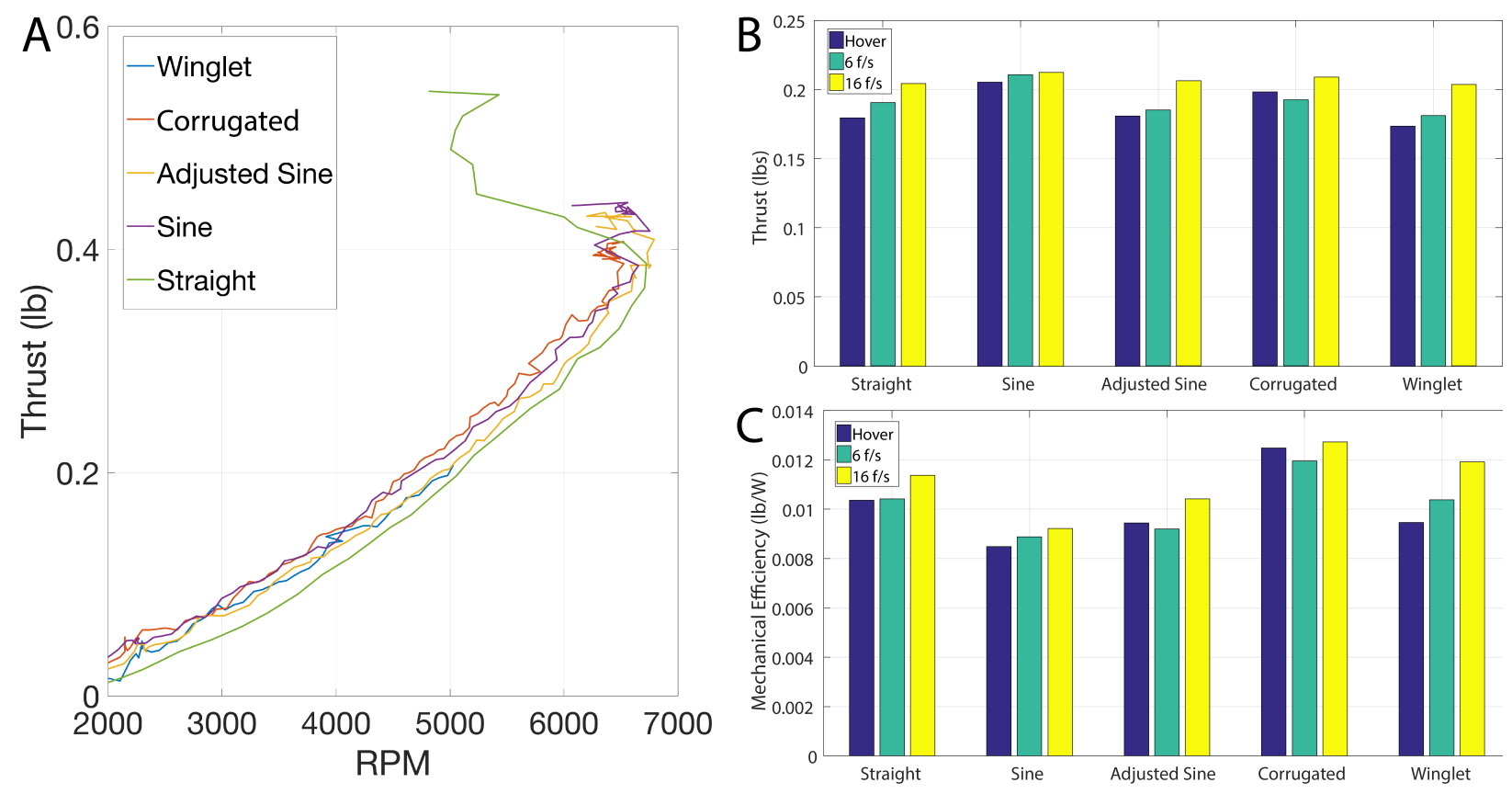

Fig. 7 Figure A shows the thrust from an RPM sweep for each of the propeller designs, all of the altered designs resulted in a greater thrust at a specified RPM than the straight blade design. Figures B and C are the thrust and mechanical efficiency at various airspeeds in the ascent configuration and a constant RPM of 4,500 RPM.

Fig. 7C shows the comparison of the mechanical efficiency between each blade design. We are defining mechanical efficiency as

$$
\eta=\frac{T}{P}
$$

where $T$ is the thrust and $P$ is the mechanical power and is determined by $P=\tau \omega$ where $\tau$ is the torque measured from the differential load cells on the thrust stand and $\omega$ is the rotor rotational speed measured from the dynamometer. We chose not to use the more typical definition of efficiency $\eta=\frac{C_{T} J}{C_{P}}$ in order to be able to provide a direct comparison to hover conditions. We can see that while the sine wave design had one of the better thrust characteristics, it came at the cost of efficiency and that the corrugated blade design had the best thrust output. As we would expect as the advanced ratio $J$ increases all of the designs increase in efficiency. 


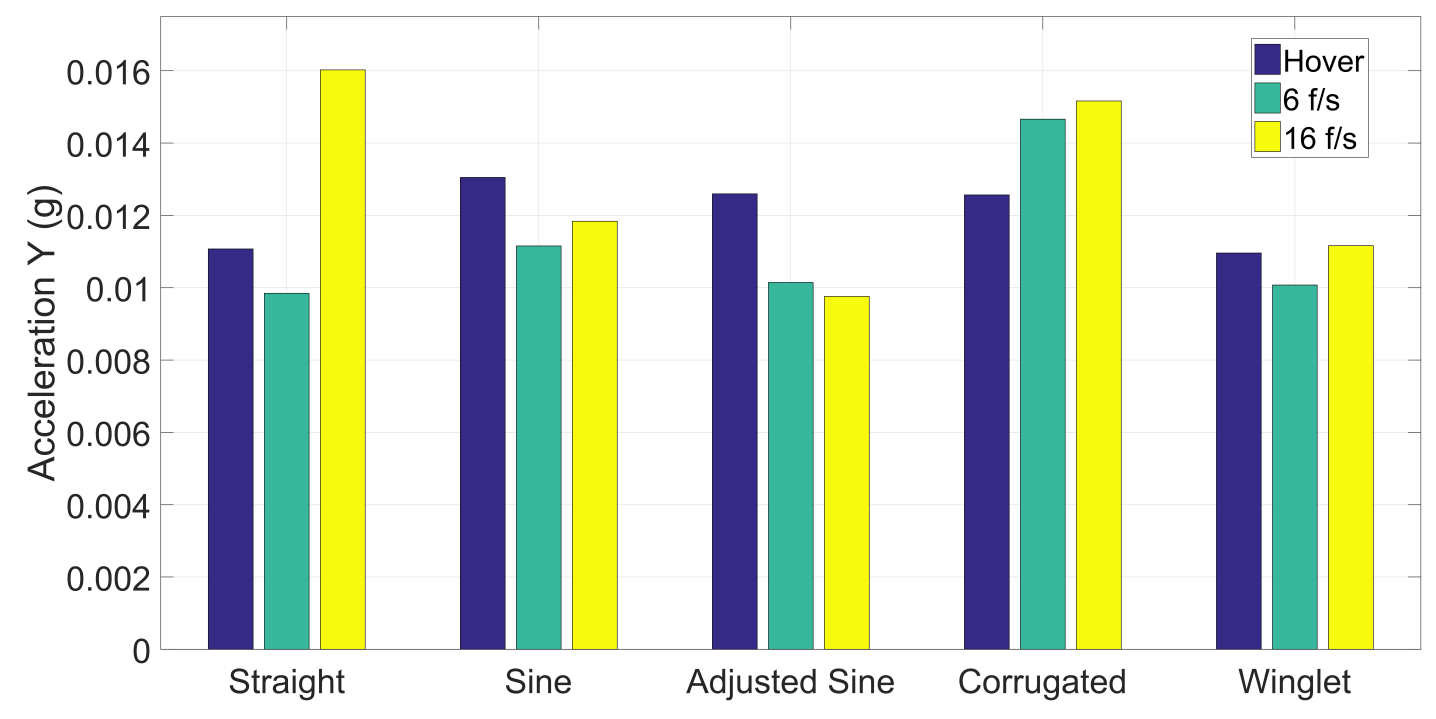

Fig. 8 The acceleration in the non-gravity direction orthogonal to thrust for various airspeeds in the ascent configuration.

One of the major concerns with and design that is asymmetric, like the anti-phase designs presented here, is the unbalanced cyclic loading that could cause premature failure due to fatigue. The experimental design and study that we are presenting here was not intended to address this issue. Figure 8 shows the acceleration in the y-direction which is the non-thrust and non-gravity direction. The acceleration should be a good measure for the effect of the unbalanced loading because it represents the vibration amplitudes of the propellers. If the system were to be completely balanced and vibrationally isolated, we would expect to see the average acceleration be zeros. This is not the case, because, if the anti-phase rotor designs were causing additional vibration, we would expect to see a large difference between the nominal straight blade and the anti-phase designs. Figure 8 shows very little variation between the straight blade, sine, adjusted sine, and winglet designs for hover and $6 \mathrm{ft} / \mathrm{s}$. The corrugated shows a larger variation from the straight blade design at hover and $6 \mathrm{ft} / \mathrm{s}$ but is closer at $16 \mathrm{ft} / \mathrm{s}$. These values are all small, and we believe that the general trend would continue as the blade size increases and also because the general trend for UAM is to have smaller blades at lower speeds with lower blade loading as described in [26]. Moreover, in this experiment, we did not try to design the anti-phase rotors to achieve dynamically balanced rotors. If dynamic balancing is a design requirement, then an anti-phase rotor can still be designed to achieve the dynamic balancing requirement either during the design or during operation by means of mass balancing.

\section{B. Acoustic Results}

With the knowledge that anti-phase trailing edge designs do not diminish the mechanical performance of the rotors, the analysis of the acoustic experimentation can move forward. The acoustic data gathered from the microphone array are aggregated and output in the frequency domain in decibels. For this study we will be using A-weighting which is a method to convert the sound pressure level into a signature that is more representative of what humans hear[27]. The conversion between unweighted sound pressure level and air pressure is

$$
S P L=10 \log _{10}\left(\frac{p}{p_{\text {ref }}}\right)
$$

where $p_{r e f}$ is atmospheric pressure at sea level. The A-weighted pressure is calculated by

$$
R_{A}(f)=\frac{12194^{2} f^{4}}{\left(f^{2}+20.6^{2}\right) \sqrt{\left(f^{2}+107.7^{2}\right)\left(f^{2}+737.9^{2}\right)}\left(f^{2}+12194^{2}\right)}
$$




$$
S P L_{A}=20 \log _{10}\left(R_{A}(f) \frac{p}{p_{\text {ref }}}\right)+2
$$

For the rest of this paper, the acoustic results will be presented in $\mathrm{dBA}$, the A-weighted sound pressure level.
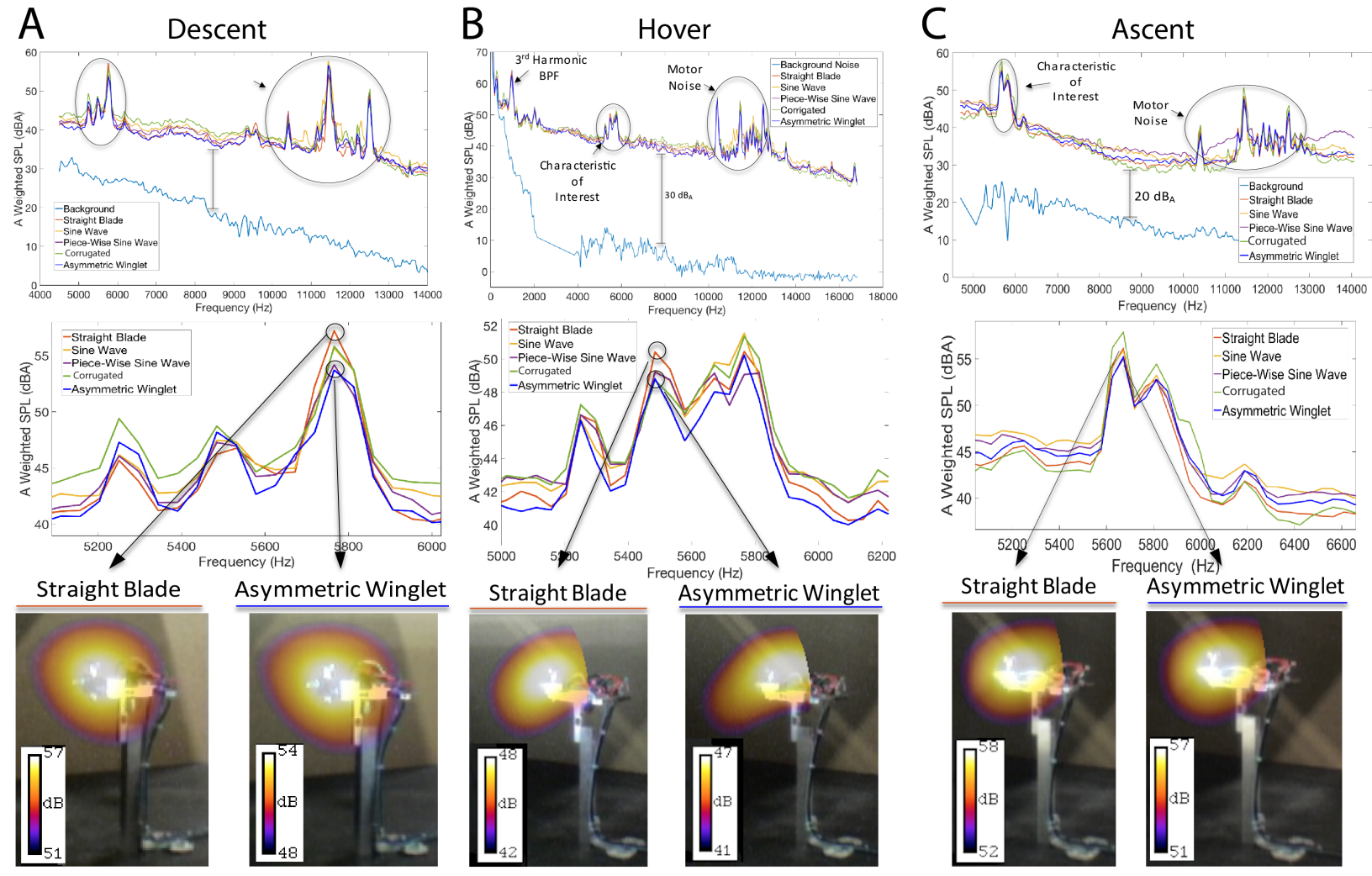

Fig. 9 Figures A, B, C show the frequency spectrum of the SPLA, the zoomed in BVI section and the spatial noise source for the peak frequency for descent, hover, and ascent respectively.

Figure 9 shows the frequency domain SPL of descent, hover, and ascent conditions at $16 \mathrm{ft} / \mathrm{s}$. The first row of Fig. 9 is the full spectrum $\mathrm{SPL}_{A}$ of each condition with the lower frequencies for the descent and ascent removed because the wind tunnel noise washed it out. These plots show that the noise of the propeller in each of the conditions is distinguishable from the background noise. In each of the plots in the first row, certain spectrum characteristics can be determined, such as the motor noise between 10,000 and $13,000 \mathrm{~Hz}$ and a characteristic of interest that is believed to be BVI between 5,000 and 6,500 Hz. We believe this to be BVI because it is at its largest in the descent condition and it is smallest in ascent conditions. The plots in the second row of Fig. 9 are the zoom-in plots to more clearly show the BVI characteristic. From this we can see that the asymmetric winglet design has the largest effect on the reduction in magnitude for this most prominent noise peak. The 24 microphones in the microphone array allow for the recreation of a spatial noise spectrum that we show in Fig. 9 where for each test condition the straight blade is compared to the asymmetric winglet. We can see that the maximum SPL is focused on the center hub in each of the conditions that peak SPL is $1 \mathrm{~dB}$ lower for the asymmetric winglet than for the straight blade design. The purple outer ring of the pressure is reduced is reduced by $1 \mathrm{~dB}$ and has a slightly reduced area contained for the anti-phase asymmetric winglet. The shape change of the spatial noise spectrum is especially pronounced in Fig. 9 $\mathrm{B}$ where the shape of the noise source is smeared by the asymmetric winglet further pushing the peak noise source downstream and supporting the stated goal of the winglets to separate the vortex interactions.

The results from Fig. 9 can be condensed by consolidating the spectrum and comparing the difference between each design as shown below.

$$
\text { Total } S P L_{A}=20 \log _{10}\left(\int_{f_{i}}^{f_{n}} R_{A}(f) \frac{p(f)}{p_{\text {ref }}} d f\right)+2
$$


where $f_{i}$ is the desired starting frequency for the summation and $f_{n}$ is the desired stopping frequency, and the comparison between the two designs is

$$
\Delta S P L_{A}=20 \log _{10}\left(\int_{f_{i}}^{f_{n}} R_{A}(f) \frac{p_{\text {anti-phase }}(f)}{p_{\text {ref }}} d f\right)-20 \log _{10}\left(\int_{f_{i}}^{f_{n}} R_{A}(f) \frac{p_{n}(f)}{p_{\text {ref }}} d f\right)
$$

where $p_{\text {anti-phase }}$ is the pressure from the anti-phase designs and $p_{n}$ is the pressure of the nominal straight blade. We are using Eqn. 6 as a comparative tool and is not intended to emulate a noise signal being removed from another noise signal but a comparison between designs.
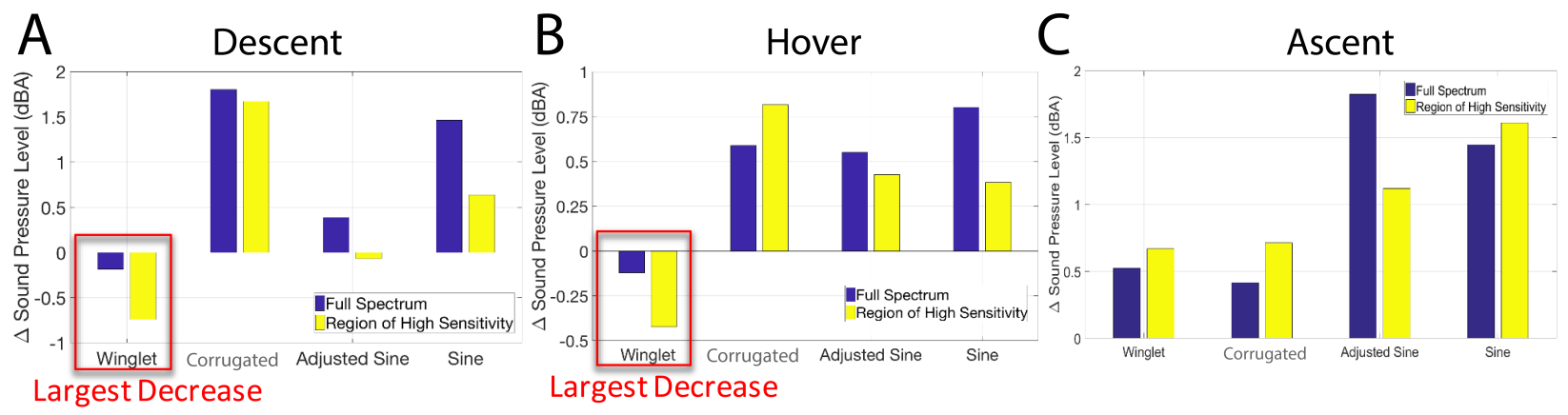

Fig. 10 The cumulative noise comparisons between the baseline straight blade design and the other designs for descent, hover, and ascent in Figures A, B, C respectively. The region of high sensitivity is from 5,000 $\mathrm{Hz}$ to 7,000 Hz.

We have split the comparisons into full spectrum and region of high sensitivity which is from 5,000 $\mathrm{Hz}$ to 7,000 $\mathrm{Hz}$ and relates to the region that humans find the most annoying. [28] The full spectrum is determined by the point when all the designs are $10 \mathrm{dBA}$ above the background noise. This means that the full spectrum measurements are not comparable between conditions. Fig. 10 shows that at 4,500 rpm the winglet is the only design that can reduce the amount of noise and does so in both the full spectrum and region of high sensitivity. None of the designs showed any performance gains in the ascent scenario. This is likely because in hover and descent the design is countering the vortex interaction by enforcing wake separation via the mechanisms shown in Figs. 3 and 4 . In the ascent mode the straight blade typically has more vortex separation due to the ascending cross flow. The anti-phase design counters this cross flow induced separation by pushing the vortices up on one blade and down on the other.

The comparisons presented in Fig. 10 do not take into account the fact that at the same RPM their are different thrust values generated by the different anti-phase designs. To help compensate we applied a normalization to the pressure by the thrust ratio between the anti-phase designs and the straight blade using

$$
\Delta \mathrm{S} \overline{\mathrm{P}} \mathrm{L}=20 \log _{10}\left(\int_{f_{i}}^{f_{n}} R_{A}(f) \frac{p_{\text {anti-phase }}(f)}{p_{\text {ref }}} d f \frac{T_{S}}{T}\right)-20 \log _{10}\left(\int_{f_{i}}^{f_{n}} R_{A}(f) \frac{p_{n}(f)}{p_{\text {ref }}} d f\right)
$$

where $T_{s}$ is the thrust of the straight blade and $T$ is the thrust of the anti-phase blade.
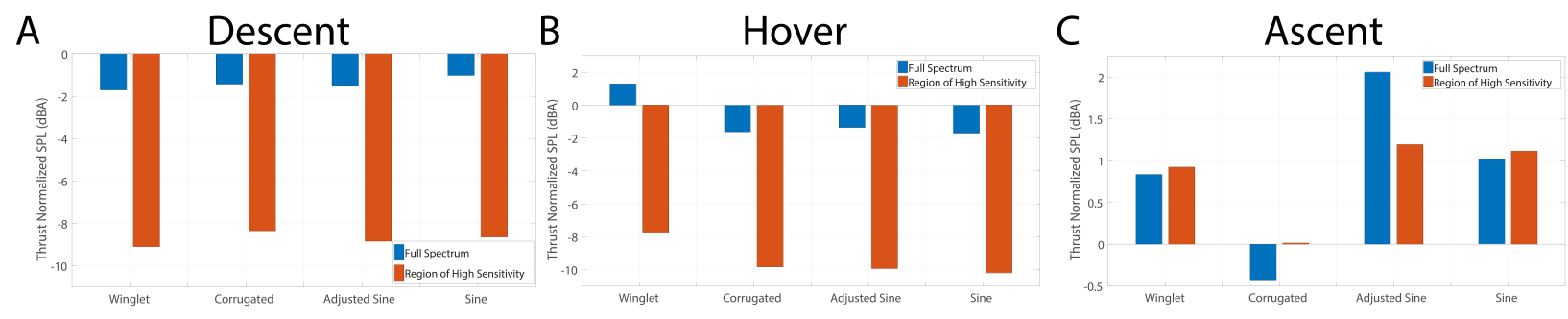

Fig. 11 The change in normalized thrust SPL between the anti-phase configurations and the straight blade are shown in Figure A, B, C for decent, hover, and ascent respectively. 
Figure 11 shows the comparison of the difference between the thrust normalized SPL and the straight blade. From Fig. 11 we can see that all of the designs faired particularly better because they were increasing the amount of thrust compared to the straight blade. We can also see that in hover the corrugated, adjusted sine, and sine anti-phase designs performed better than the winglet. The ascent shows some small gains for the corrugated which was already the best performing of the designs in Fig. 10 C. Theses values are meant to demonstrate the there are noise differences between the blades due to the loading noise and are not intended to necessarily be predictive.

divide the noise by the amount of thrust being produced. From this metric we can see that the corrugated has the lowest amount of noise per pound of thrust in descent and ascent. In hover it is the sine design but corrugated is close to the sine design in hover. This suggests that as the necessary thrust increases the amount of noise generated by the corrugated design will be proportionally smaller compared to the nominal blade.

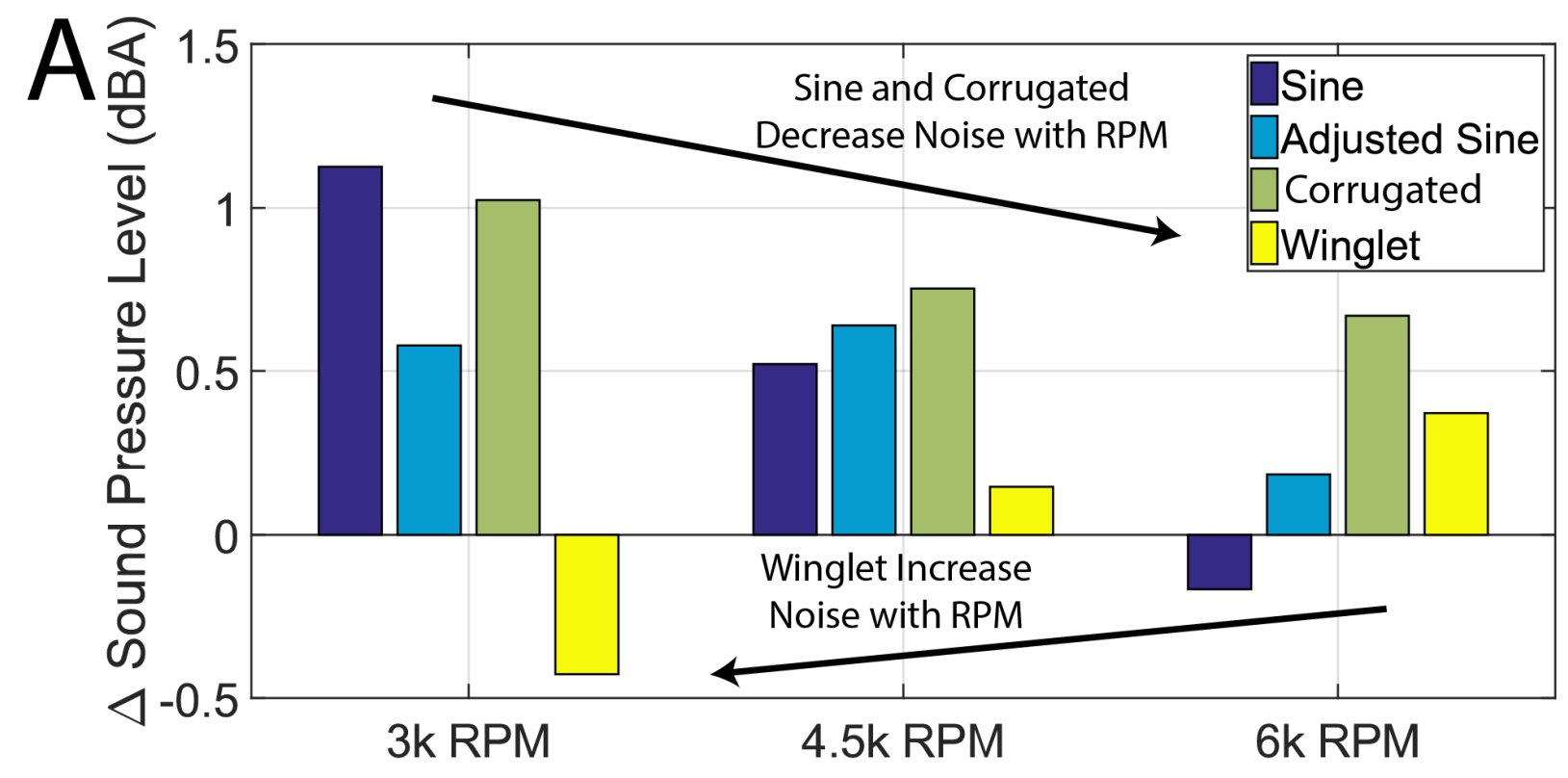

B

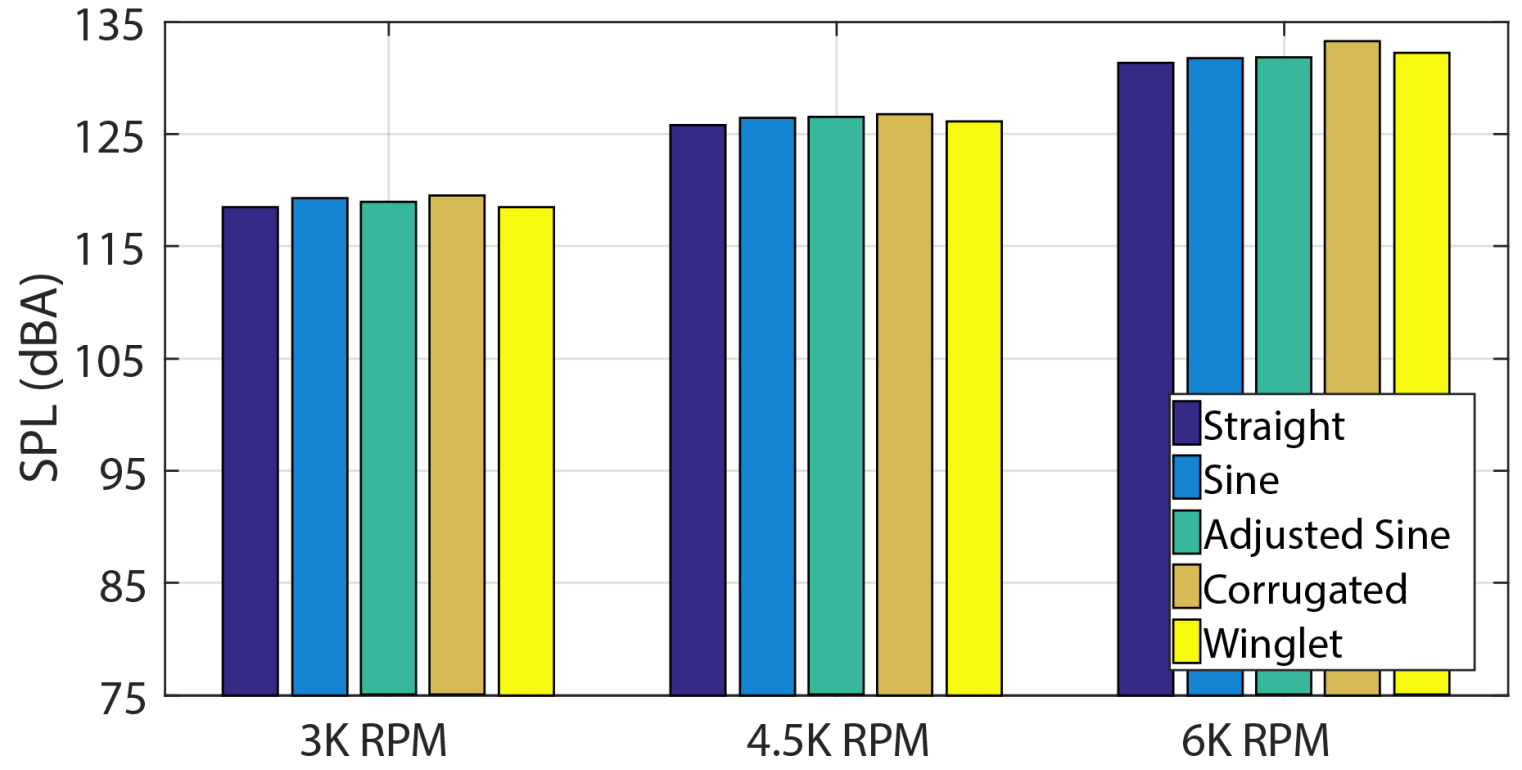

Fig. 12 Figure A shows the variation in performance for the designs with RPM. The winglets performance decreases with increases in RPM whereas the other designs trend the other direction increasing their relative performance with RPM. Figure B shows that the jumps in RPM dwarf the differences between designs. 
Figure 12 shows the comparison between the different designs at different RPMs in hover. The sine and corrugated designs decrease their relative noise as the RPM increases whereas the asymmetric winglet has the opposite trend. This supports the observations from Figure 11 where the corrugated and sine designs had the smallest dBA per thrust values. The adjusted sine design does not follow the same trend as the sine and corrugated designs at 4,500 rpm The asymmetric winglet design probably displays this pattern because at lower speeds it is less likely to have its trailing blade intersect the tight tip vortex core shed by the preceding blade with the upward swept winglet. On the other hand, the other designs probably have a sweet spot where their spatial pattern matches with the rotational frequency.

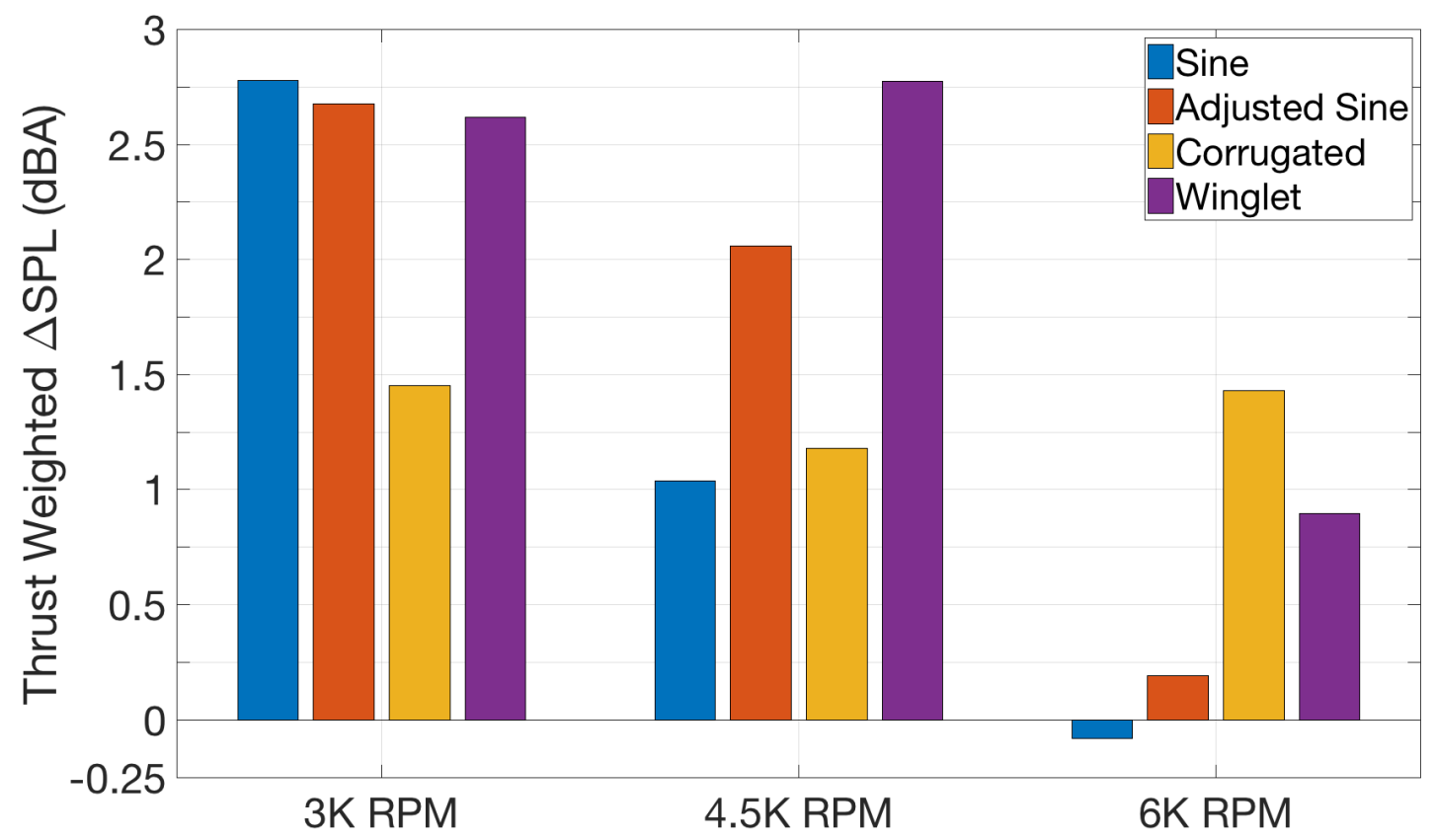

Fig. 13 The noise per thrust decreases as the absolute amount of noise is increasing with RPM as shown in Fig. 12

Figure 13 shows the comparison of the change in noise normalized with respect to thrust at different RPMs in hover. We can see that the much like Fig. 12A the sine wave continues to perform better as the RPM increases, as does the adjusted sine. The asymmetric winglet design though shows that it's performance adjusted per thrust is best at the highest RPM which is the opposite effect seen in Fig. [12. This suggests that asymmetric winglet design begins producing more thrust at higher RPMs to compensate for the amount of extra noise it's producing. The corrugated design shows a minimum in performance at $4.5 \mathrm{~K}$ RPM for thrust weighted change in noise suggesting at a minimum value is reached somewhere between $3 \mathrm{~K}$ RPM and 4.5K RPM.

It is worth noting that in between the experiments that generated the data for Figs. 9 - 13 the winglet and sine wave propellers were replaced as was the motor. We can see that the magnitudes of the plots in Fig. 10B, and Fig. 12 do not match perfectly and are notably different in the sine and winglet designs. These differences reflect the importance of uncertainty sources in the experiment which exist not only in the measurement and post-test data processing but also in the 3D printing since the replacement propellers could be of slightly different geometries. Thus, it is important to recognize that the relative merits discerned from these figures should be viewed in light of the experimental uncertainties. 

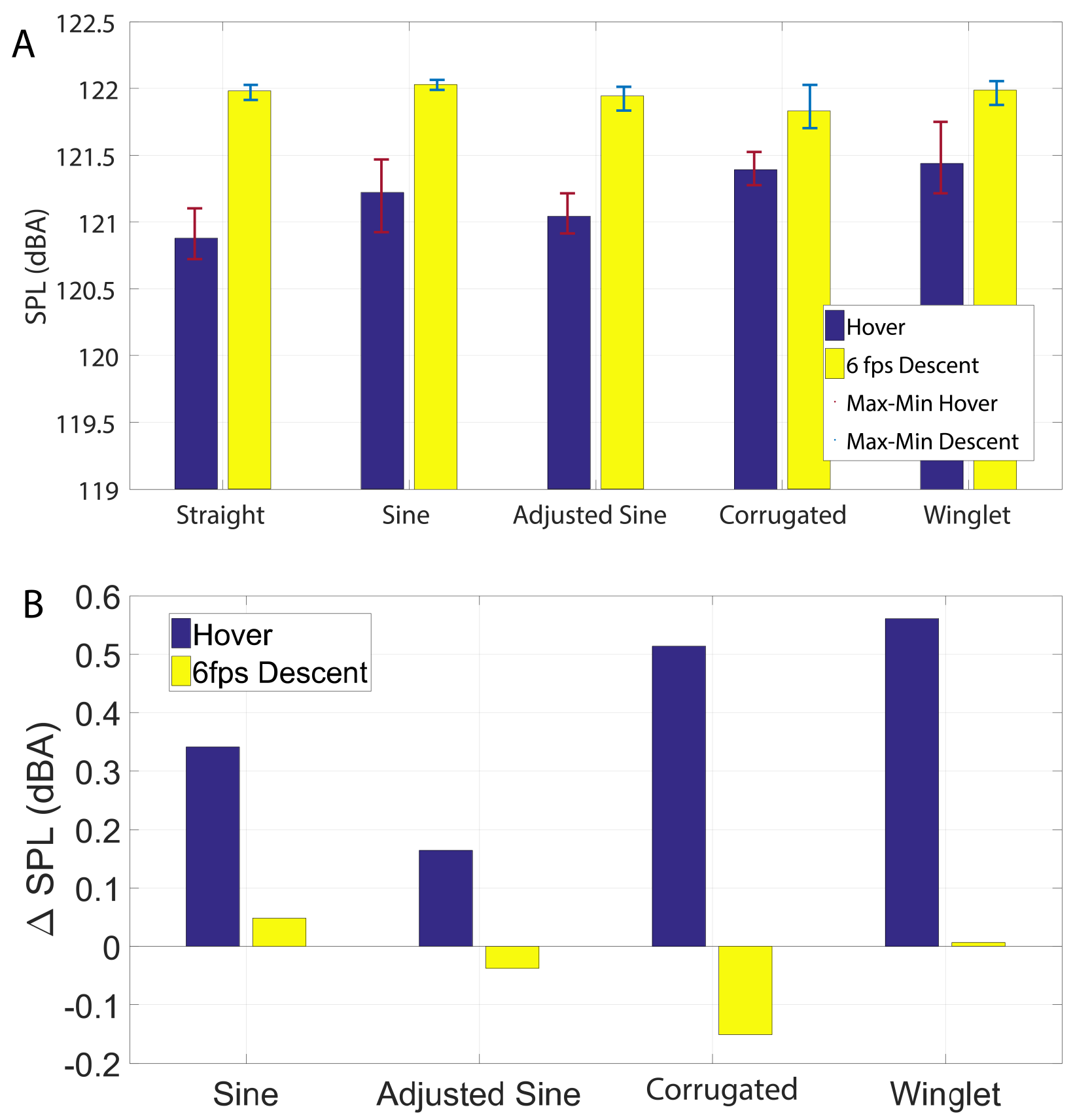

Fig. 14 Figure A shows the range of results for $0.2 \mathrm{lb}$ thrust control designs and figure $B$ shows the relative performance of the average values.

It should be further noted that these experiments are not performed in an anechoic chamber and the delta values are relatively small, so it is of critical importance to determine the repeatability of the experimental set-up. The repeatability studies we performed as constant thrust experiments as opposed to the constant rotational speed experiments presented earlier in this paper. The thrust controlled PI controller showed an average steady state error of $0.44 \%$ and a maximum steady state error of $3 \%$ at the commanded $0.2 \mathrm{lb}$. Each test configuration was run three times, with the propeller and tunnel being brought down between each run. The averaged in the log calculation as shown below is for the full frequency range. 


$$
\text { Average } S P L_{A}=20 \log _{10}\left(\frac{1}{3} \sum_{j=1}^{3} \int_{i}^{n} \frac{p(f)}{p_{\text {ref }}} d f\right)+2
$$

Figure 14 A shows the comparison between the SPL for each design with error bars for the maximum and minimum SPL values for hover and descent. The largest range belonged to the winglet with a range of approximately $0.55 \mathrm{dbA}$ in hover. At $6 \mathrm{ft} / \mathrm{s}$ descent the largest range belonged to the corrugated at $0.32 \mathrm{dBA}$. Figure $14 \mathrm{~B}$ does show that the corrugated and adjusted sine have small gains in descent but they are all within the max-min range from Fig. 14A.
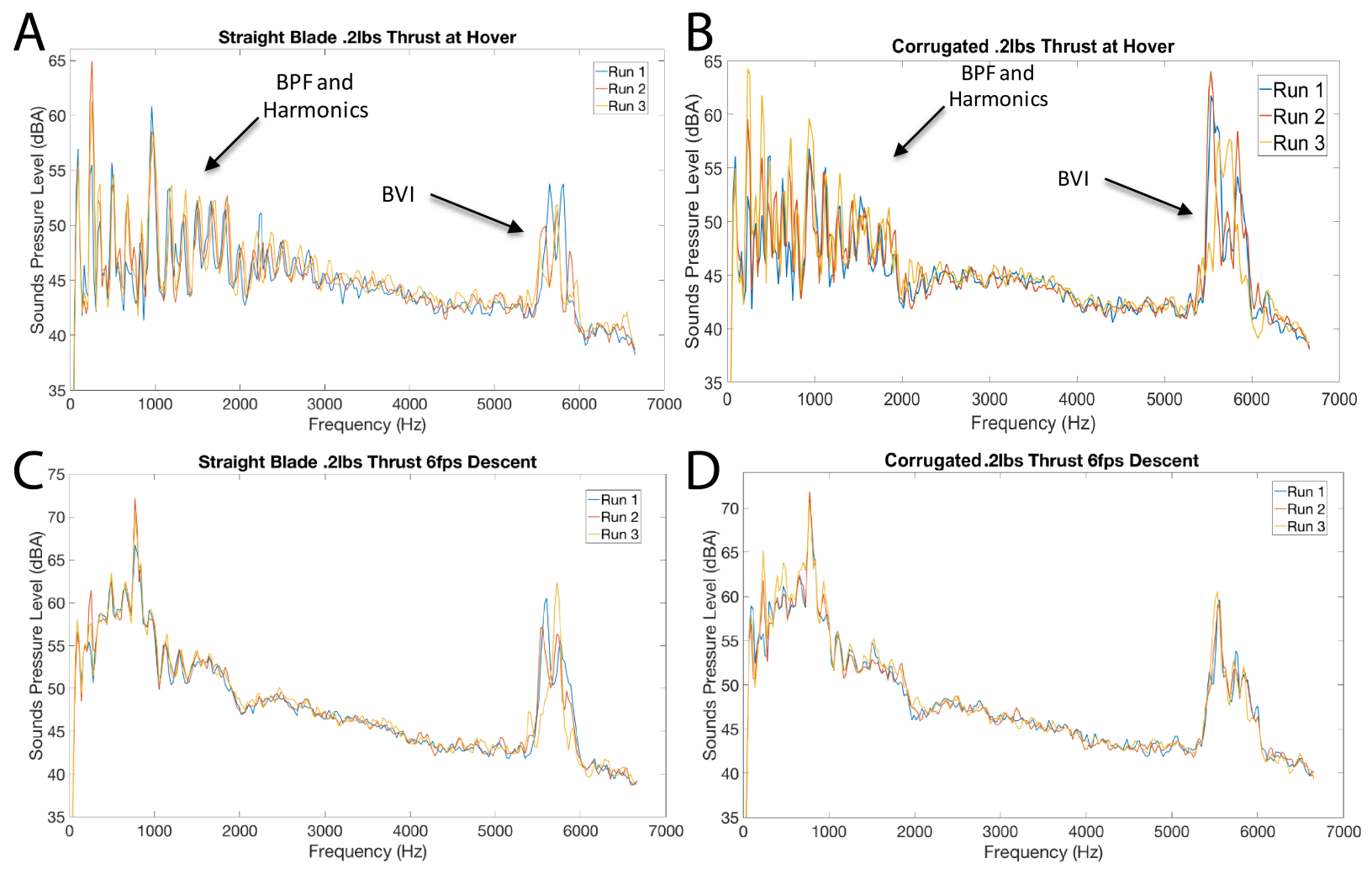

Fig. 15 Figures $\mathrm{A}$ and $\mathrm{C}$ show the multiple runs in the frequency domain for the straight blade for $0.2 \mathrm{lb}$ thrust control in hover and $6 \mathrm{ft} / \mathrm{s}$. Figures $B$ and $D$ show the same hover and descent but for the corrugated design.

Figure 15 shows the frequency domain plots for the straight blade and corrugated for hover and decent. While the total SPL has a reasonably broad range between the minimum and maximum values, the frequency plots are fairly repeatable in each set of runs. For the corrigated blade run number three had a few blade passing frequencies that exceeded the values of the BPF of runs 1-2. This variation of the peak BPF values between runs 1-2 and run 3 contributed to the variation in the experiment seen in Fig. 14. These variations could be the result of the frequency resolution of collected data, where small shifts around the peaks can result in larger deltas due to the discrete measurement jumps. Figures $15 \mathrm{C}$ and D comparatively show why the variation in the SPL values in Fig. 14 is a smaller for the descent configuration than the hover. This is because the increase in the background noise due to the wind tunnel results in a flatter profile with the harmonics being less pronounced which reduces the variation. They also demonstrate why the corrugated design performed better in Fig. 14 B because the corrugated design dramatically reduces the secondary BVI peak at around $5,800 \mathrm{~Hz}$.

The $0.2 \mathrm{lb}$ thrust control yielded little performance increases this is likely because the RPM was somewhere between $4.8 \mathrm{~K}$ and $5 \mathrm{~K}$ depending on the blade design which from Fig. $12 \mathrm{~A}$ is not high enough to being seeing advantages from the sine and too large for the asymmetric winglet. The thrust control for $0.15 \mathrm{lb}$ shown in Fig. 16] shows performance gains outside of the min-max range shown in Fig. 14. The min-max ranges from Fig. 14 act as bounds for the $0.15 \mathrm{lb}$ experiments which have less variance than the $0.2 \mathrm{lb}$. Figure 16 shows performance gains across the board which can be 
explained by Fig. 13 . The expectation based on figure 12, is that as speed decreases the relative performance of the corrugated design would decrease. However, this is true under RPM controlled scenarios, when the thrust is controlled the RPM of the higher thrust blades have a lower RPM for the same thrust as shown in Fig. 7 The reduction in RPM, in thrust control, reduces the rotational noise and combines with the BVI noise reduction. The performance differences between the thrust control at $0.2 \mathrm{lbs}$ and $0.15 \mathrm{lbs}$ are likely because $0.15 \mathrm{lbs}$ is in a sweet spot where the RPM range is between $4 \mathrm{~K}$ and $4.3 \mathrm{~K}$ for the blade designs which is low enough for the asymmetric winglets to still be gaining performance but high enough for the sine and adjusted sine to start having gains due to the compensating thrust pattern shown in Fig. 13. For the corrugated design the $0.15 \mathrm{lb}$ control is at about 4K RPM which based off Fig. 13 is likely a range where the noise compensation due to thrust is at it's greatest. All of this suggest that we begin to see diminishing acoustic reduction well before mechanical thrust saturation and there is likely an optimal spot in the RPM envelope where the acoustic reduction is the greatest. This also suggests that a design optimization of an anti-phase rotor could potentially maximize the acoustic reduction to match the peak rotor thrust. In our experiment, the anti-phase rotors were designed and 3D printed without any analytical models to guide the design due to the lack of time.

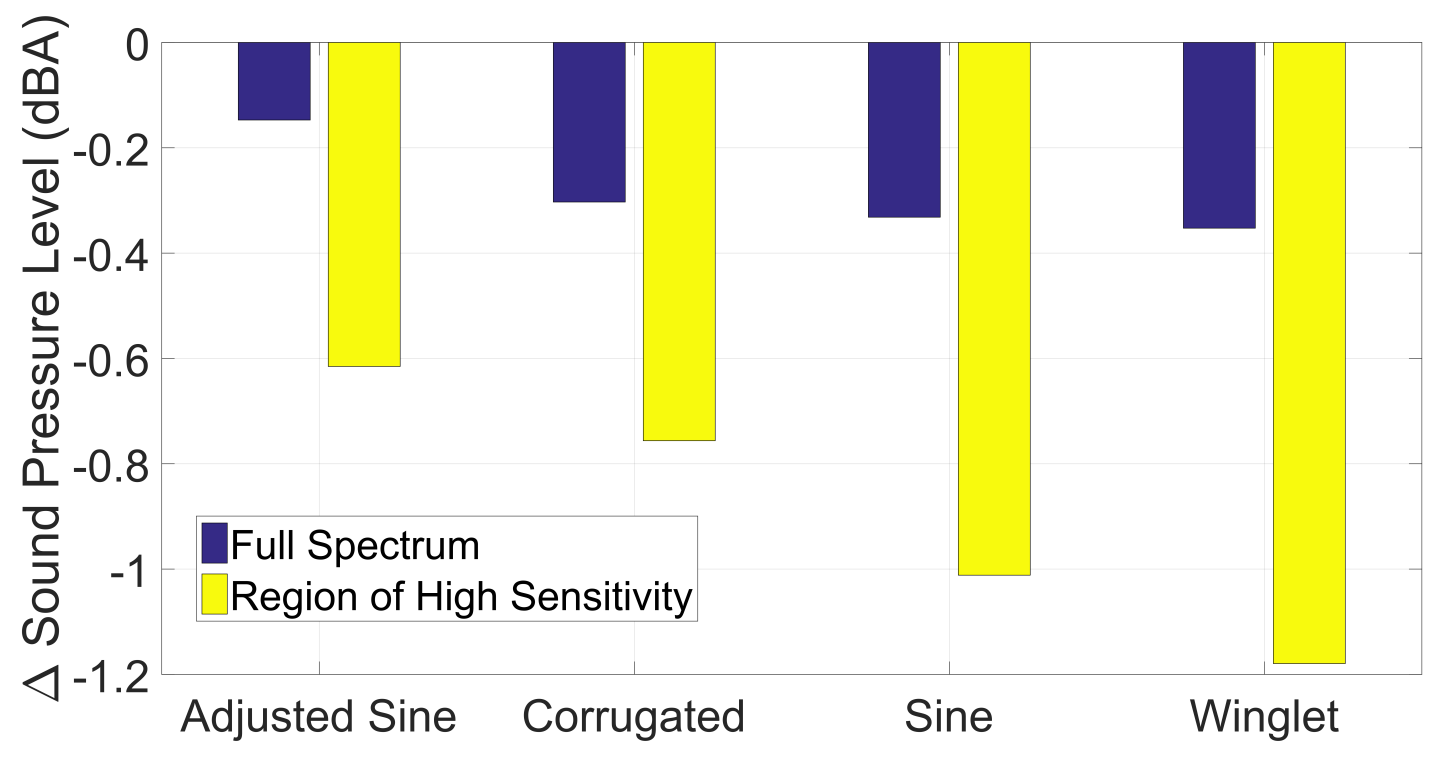

Fig. 16 Comparison of $0.15 \mathrm{lb}$ thrust controlled $\triangle S P L$ shows that the aerodynamic benefits aid in the overall noise benefits and that there is a sweet spot for thrust much like there was for RPM.

Collectively, the results suggest that all of the proposed designs are capable of reducing BVI noise, but that the acoustic gains cannot be solely captured through their vortex interactions. The fixed RPM experiments combined with the fixed thrust experiments suggest that when RPM is fixed the vortex and BVI noise is decreased but the loading noise is increased because of the more efficient operation of the blades. The number of different trends and the intersections of them suggests a complex design manifold that is only partially represented in this study.

While even the best noise reductions shown here have only shown a reduction in noise of approximately $1 \mathrm{dBA}$, this still could yield significant benefits. Consider the UAM scenario presented above with the target of $65 \mathrm{dBA}$ at a distance of $250 \mathrm{ft}$. If we assume an undisturbed sphere of air for that noise to propagate from we can assume that the energy remains constant and further assuming that the energy remains constant, then we can determine

$$
\Delta r=r_{\text {init }}\left(1-10^{\frac{S P L_{\text {reduction }}}{20}}\right)
$$

derived from the geometric propagation of sound pressure level from [29]. This results in a change of just over 27 feet or a reduction of $10.8 \%$ of the radius of operation for a $1 \mathrm{dBA}$ noise reduction. These are, of course, ideal numbers, we are not attempting to acoustically scale, just comparing the magnitudes. Additionally, the actual implementation would have acoustic interference between the rotors and aircraft body, but it shows the importance of small gains in a small-scale rotor experiment which could translate into more significant gains in a full-scale rotor. 


\section{Conclusions and Future Work}

In this paper, we have presented several innovative anti-phase rotor noise reduction concepts. These concepts include the anti-phase alternating trailing edge patterns with varying phase shift from blade to blade and an asymmetric blade tip concept. The anti-phase trailing edge concepts effectively create a non-planar wake that could reduce the blade vortex interaction which in turn could lead to noise reduction. The asymmetric blade tip concept has been shown in high fidelity CFD simulations that it could be capable of reducing the harmonic reinforcement of blade tip vortices.

To assess the potential acoustic reduction benefits of the proposed anti-phase rotor technologies, we conducted an experimental investigation on four different anti-phase rotors at constant thrust and constant RPM in the descent, ascent, and hover mode. The test results indicate that the proposed anti-phase rotor concepts appear to be an effective means of reducing blade vortex interaction noise. The results also suggest that there appears to be an optimal RPM at which the acoustic reduction benefits of the tested anti-phase rotors appear to be the greatest. This is hardly a surprise since the tested anti-phase rotors were not optimized in the design. With design optimization using analytical acoustic prediction capabilities of computational tools, it is expected that an anti-phase rotor design could provide the maximum noise reduction at a matching peak thrust requirement.

There are many implications resulting from the use of the proposed anti-phase rotor designs. One implication is unbalanced loading which could result in operational issues that would require methods or means for achieving dynamic balancing either in the design or in field operations. If vibration remains an issue because of the unbalanced loading even with dynamic balancing, there could be additional needs for active control compensation and or altered maintenance schedules. Another implication is the possibility that different anti-phase blades could be designed for different flight path operations or that the vehicle flight path planning could be altered to take advantage of acoustic directionality when the anti-phase rotor designs are used in conjunction with a two-axis tilt rotor design. In future work, we would like to investigate further the possibilities mentioned above and to test the anti-phase rotor designs on larger rotors in a more controlled environment such as an anechoic chamber. If time is not a constraint in an ideal setting, developing analytical models for acoustic prediction should be performed in conjunction with experimental investigations in order to better understand the noise reduction mechanisms so that the anti-phase rotor designs can be optimized to better take advantage of the physical insight gained from the analytical models.

\section{Acknowledgments}

The authors would like to thank the personnel of NASA Ames Fluid Mechanic Laboratory and particularly Dr. Rabindra Mehta for their experimental support. The authors also would like to thank the personnel of the Computational Aerosciences Branch and particularly Dr. Cetin Kiris and Dr. Jeffrey Housman for their CFD support. This research is funded by NASA Aeronautics Research Mission Directorate (ARMD) Convergent Aeronautics Solutions (CAS) project.

\section{References}

[1] Moore, M. D., "Personal air vehicles: a rural/regional and intra-urban on-demand transportation system," Journal of the American Institute of Aeronautics and Astronautics (AIAA), Vol. 2646, 2003.

[2] Duffy, M. J., Wakayama, S. R., and Hupp, R., "A Study in Reducing the Cost of Vertical Flight with Electric Propulsion," 17th AIAA Aviation Technology, Integration, and Operations Conference, 2017, p. 3442.

[3] Antcliff, K. R., Moore, M. D., and Goodrich, K. H., "Silicon Valley as an Early Adopter for On-Demand Civil VTOL Operations," 16th AIAA Aviation Technology, Integration, and Operations Conference, 2016, p. 3466.

[4] “Volocopter VC2," https://wwW.volocopter.com/assets/pdf/2017_04_Design_specifications_2X.pdf. 2017. Accessed: 2018-06-3.

[5] Wright, S., "The acoustic spectrum of axial flow machines," Journal of Sound and Vibration, Vol. 45, No. 2, 1976, pp. 165-223.

[6] Kurtz, D., and Marte, J., “A review of aerodynamic noise from propellers, rotors, and lift fans,” 1970.

[7] Splettstoesser, W., Kube, R., Wagner, W., Seelhorst, U., Boutier, A., Micheli, F., Mercker, E., and Pengel, K., "Key results from a higher harmonic control aeroacoustic rotor test (HART)," Journal of the American Helicopter Society, Vol. 42, No. 1, 1997, pp. 58-78.

[8] Brocklehurst, A., and Barakos, G., "A review of helicopter rotor blade tip shapes," Progress in aerospace sciences, Vol. 56, 2013, pp. 35-74. 
[9] Yung, H. Y., "Rotor blade-vortex interaction noise," Progress in Aerospace Sciences, Vol. 36, No. 2, 2000 , pp. 97-115.

[10] Yang, S. J., Baeder, J. D., et al., "Aerodynamics and Aeroacoustics of Spanwise Wavy Trailing Edge Flatback Airfoils: Design Improvement," North American Wind Energy Academy 2015 Symposium, Virginia Tech, 2015.

[11] Hardin, J. C., and Lamkin, S. L., "Concepts for reduction of blade/vortex interaction noise," Journal of Aircraft, Vol. 24, No. 2, 1987, pp. 120-125.

[12] Tauszig, L., and Gandhi, F., "Influence of blade-to-blade dissimilarity on alleviation of helicopter blade-vortex interactions," Mathematical and computer modelling, Vol. 33, No. 10-11, 2001, pp. 1139-1154.

[13] Chen, P. C., Baeder, J. D., Evans, R. A., and Niemczuk, J., "Blade-vortex interaction noise reduction with active twist smart rotor technology," Smart Materials and structures, Vol. 10, No. 1, 2001, p. 77.

[14] Gautreau, J. C., and Wassynger, S. P., "Method of providing non-uniform stator vane spacing in a compressor,”, Jun. 292010. US Patent 7,743,497.

[15] Nguyen, N., Guist, R., and Muzzio, D., "Experimental investigation of the rotor blade vibration in the three-stage compressor of the 11-by 11-Foot Transonic Wind Tunnel," 31st Joint Propulsion Conference and Exhibit, 1995, p. 3139.

[16] Nguyen, N., “Anti-Phase Vortex Reduction Control for Rotor Noise Suppression,” Proposal to NASA Ames Center Innovation Funds (CIF) Program, 2018.

[17] Nguyen, N., "Elastically shaped future air vehicle concept,” NASA Innovation Fund Award, 2010.

[18] Nguyen, N., and Urnes, J., "Aeroelastic modeling of elastically shaped aircraft concept via wing shaping control for drag reduction," AIAA Atmospheric Flight Mechanics Conference, 2012, p. 4642.

[19] Panzino, C., ????

[20] Ting, E., Chaparro, D., Nguyen, N., and Fujiwara, G. E., "Optimization of Variable-Camber Continuous Trailing-Edge Flap Configuration for Drag Reduction,” Journal of Aircraft, 2018, pp. 1-23.

[21] “8x3.8SF," https://www. apcprop.com/product/8x3-8sf/ 2017. Accessed: 2018-06-12.

[22] J.B. Brandt, G. A., R.W. Deters, and Selig, M., “UIUC Propeller Database," http://m-selig.ae.illinois.edu/props/ propDB.html, 2015. Accessed: 2018-6-9.

[23] Mueller, R., "The influence of winglets on rotor aerodynamics," 1986.

[24] Schneider, C. A., Rasband, W. S., and Eliceiri, K. W., "NIH Image to ImageJ: 25 years of image analysis," Nature methods, Vol. 9, No. 7, 2012, p. 671.

[25] “Series 1580 Thrust Stand and Dynamometer," https://www.rcbenchmark.com/pages/series-1580-thrust-standdynamometer. 2018. Accessed: 2018-06-12.

[26] Johnson, W., Silva, C., and Solis, E., "Concept Vehicles for VTOL Air Taxi Operations,” AHS International Technical Meeting on Aeromechanics Design for Transformative Vertical Flight, San Francisco, CA, 2018.

[27] IEC, I., “61672: 2003: Electroacoustics-Sound Level Meters,” Tech. rep., Technical Report, IEC, 2003.

[28] Kumar, S., von Kriegstein, K., Friston, K., and Griffiths, T. D., "Features versus feelings: dissociable representations of the acoustic features and valence of aversive sounds," Journal of Neuroscience, Vol. 32, No. 41, 2012, pp. 14184-14192.

[29] Crocker, M. J., Handbook of acoustics, John Wiley \& Sons, 1998. 